\title{
A Game-Theoretic Approach to Energy-Efficient Power Control in Multicarrier CDMA Systems
}

\author{
Farhad Meshkati, Student Member, IEEE, Mung Chiang, Member, IEEE, H. Vincent Poor, Fellow, IEEE, and \\ Stuart C. Schwartz, Life Fellow, IEEE
}

\begin{abstract}
A game-theoretic model for studying power control in multicarrier code-division multiple-access systems is proposed. Power control is modeled as a noncooperative game in which each user decides how much power to transmit over each carrier to maximize its own utility. The utility function considered here measures the number of reliable bits transmitted over all the carriers per joule of energy consumed and is particularly suitable for networks where energy efficiency is important. The multidimensional nature of users' strategies and the nonquasi-concavity of the utility function make the multicarrier problem much more challenging than the single-carrier or throughput-based-utility case. It is shown that, for all linear receivers including the matched filter, the decorrelator, and the minimum-mean-square-error detector, a user's utility is maximized when the user transmits only on its "best" carrier. This is the carrier that requires the least amount of power to achieve a particular target signal-to-interference-plus-noise ratio at the output of the receiver. The existence and uniqueness of Nash equilibrium for the proposed power control game are studied. In particular, conditions are given that must be satisfied by the channel gains for a Nash equilibrium to exist, and the distribution of the users among the carriers at equilibrium is characterized. In addition, an iterative and distributed algorithm for reaching the equilibrium (when it exists) is presented. It is shown that the proposed approach results in significant improvements in the total utility achieved at equilibrium compared with a single-carrier system and also to a multicarrier system in which each user maximizes its utility over each carrier independently.
\end{abstract}

Index Terms-Energy efficiency, game theory, multicarrier code-division multiple-access (CDMA), multiuser detection, Nash equilibrium, power control, utility function.

\section{INTRODUCTION}

$\mathbf{P}$ OWER CONTROL is used for resource allocation and interference management in both the uplink and downlink of code-division multiple-access (CDMA) systems. In the uplink, the purpose of power control is to allow each user to transmit enough power so that it can achieve the required quality-ofservice (QoS) at the uplink receiver without causing unnecessary interference to other users in the system. One of the key issues in wireless system design is energy consumption at users' terminals. Since in many scenarios, the users' terminals

Manuscript received April 1, 2005; revised October 1, 2005. This research was supported in part by the National Science Foundation under Grant ANI-0338807, Grant CNS-04-27677, Grant CNS-04-17607, and Grant CCF-04-48012. This work was presented in part at the 2005 IEEE Wireless Communication and Networking Conference, New Orleans, LA, March 14-17, 2005.

The authors are with the Department of Electrical Engineering, Princeton University, Princeton, NJ 08544 USA (e-mail: meshkati@princeton.edu; chiangm@princeton.edu; poor@ princeton.edu; stuart@ princeton.edu).

Digital Object Identifier 10.1109/JSAC.2005.864028 are battery-powered, efficient energy management schemes are required in order to prolong the battery life. Hence, power control plays an even more crucial role in such systems. Recently, game theory has been used to study power control in data networks and has been shown to be a very effective tool for examining this problem (see, for example, [1]-[9]). In [1], the authors provide some motivation for using game theory to study communication systems, and in particular power control. In [2] and [3], power control is modeled as a noncooperative game in which users choose their transmit powers in order to maximize their utilities, where utility is defined as the ratio of throughput to transmit power. In [4], pricing is introduced to obtain a more efficient solution. Similar approaches are taken in [5]-[8] for different utility functions. In [9], the authors extend the approach in [2] to study the cross-layer problem of joint multiuser detection and power control.

Multicarrier CDMA, which combines the benefits of orthogonal frequency-division multiplexing (OFDM) with those of CDMA, is considered to be a potential candidate for next-generation high data-rate wireless systems (see [10]). In particular, in multicarrier direct-sequence CDMA (DS-CDMA), the data stream for each user is divided into multiple parallel streams. Each stream is first spread using a spreading sequence and is then transmitted on a carrier [11]. In a single-user scenario with a fixed total transmit power, the optimal power allocation strategy for maximizing the rate is waterfilling over the frequency channels [12]. The multiuser scenario is more complicated. In [13]-[15], for example, several waterfilling type approaches have been investigated for multiuser systems to maximize the overall throughput. However, there are many practical situations where enhancing power efficiency is more important than maximizing throughput. For such applications, it is more important to maximize the number of bits that can be transmitted per joule of energy consumed rather than to maximize the throughput.

Consider a multiple-access multicarrier DS-CDMA network where each user wishes to locally and selfishly choose its transmit powers over the carriers in such a way as to maximize its own utility. However, the strategy chosen by a user affects the performance of other users in the network through multiple-access interference. There are several questions to ask concerning this interaction. First of all, what is a reasonable choice of a utility function that measures energy efficiency in a multicarrier network? Second, given such a utility function, what strategy should a user choose in order to maximize its utility? If every user in the network selfishly and locally picks its utility-maximizing strategy, will there be a stable state at 
which no user can unilaterally improve its utility (Nash equilibrium)? If such a state exists, will it be unique? What will be the distribution of users among the carriers at such an equilibrium?

Because of the competitive nature of the users' interaction, game theory is the natural framework for modeling and studying such a power control problem. This work is the first game-theoretic treatment of power control in multicarrier CDMA systems. We propose a noncooperative power control game in which each user seeks to choose its transmit power over each carrier to maximize its overall utility. The utility function here is defined as the ratio of the user's total throughput to its total transmit power over all the carriers. This utility function, which has units of bits/joule, measures the total number of reliable bits transmitted per joule of energy consumed and is particularly suitable for applications where saving power is critical. Because of the noncooperative nature of the proposed game, no coordination among the users is assumed. Compared with prior work on noncooperative power control games, there are two difficulties to the problem studied in this paper. One is that users' strategies in the multicarrier case are vectors (rather than scalars) and this leads to an exponentially larger strategy set for each user (i.e., many more possibilities). Second, the energy efficiency utility function, which is considered here, is nonquasi-concave. This means that many of the standard theorems from game theory as well as convex optimization cannot be used here. In this work, we derive the Nash equilibrium [16] for the proposed power control game and study its existence and uniqueness. We also address the following questions. If there exists a Nash equilibrium for this game, can the users reach the equilibrium in a distributive manner? What kind of carrier allocations among the competing users will occur at a Nash equilibrium? Will there be an even spread of usage of the carriers among users? How does the performance of this joint maximization of utility over all the carriers compare with that of an approach where utility is maximized independently over each carrier? How does a multicarrier system compare with a single-carrier system in terms of energy efficiency?

The rest of this paper is organized as follows. In Section II, we provide some background for this work by discussing the power control game for the single-carrier case. The power control game for multicarrier systems is presented in Section III. The Nash equilibrium and its existence for the proposed game are discussed in Sections IV and V, respectively. In particular, in Section IV, we derive the utility-maximizing strategy for a user when all the other users' transmit powers are fixed. In Section V, we show that depending on the channel gains, the proposed power control game may have no equilibrium, a unique equilibrium, or more than one equilibrium, and we derive conditions that characterize the existence and uniqueness of Nash equilibrium for a matched filter (MF) receiver. The case of two-carrier systems is studied in more detail in Section VI, where we obtain explicit expressions for the probabilities corresponding to occurrence of various possible Nash equilibria. In Section VII, we present an iterative and distributed algorithm for reaching the Nash equilibrium (when it exists). Numerical results are presented in Section VIII. We show that at Nash equilibrium, with a high probability, the users are evenly distributed among the carriers. We also demonstrate that our proposed method of jointly

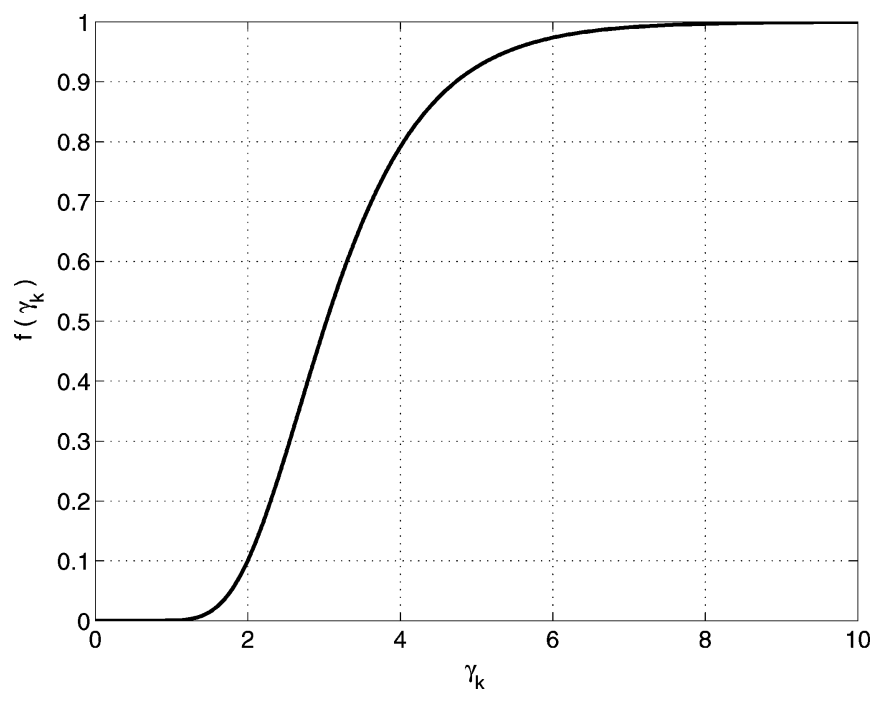

Fig. 1. A typical efficiency function (single-carrier case) representing the packet success probability as a function of received SINR.

maximizing the utility over all the carriers provides a significant improvement in performance compared with a single-carrier system, as well as the multicarrier case in which each user simply optimizes over each carrier independently. Finally, conclusions are given in Section IX.

\section{Power Control Games IN Single-Carrier Networks}

Let us first look at the power control game with a single carrier. To pose the power control problem as a noncooperative game, we first need to define a utility function suitable for data applications. Most data applications are sensitive to error but tolerant to delay. It is clear that a higher signal-to-interference-plus-noise ratio (SINR) level at the output of the receiver will generally result in a lower bit-error rate, and hence higher throughput. However, achieving a high SINR level requires the user terminal to transmit at a high power, which in turn results in low battery life. This tradeoff can be quantified (as in [2]) by defining the utility function of a user to be the ratio of its throughput to its transmit power, i.e.,

$$
u_{k}=\frac{T_{k}}{p_{k}} \text {. }
$$

Throughput is the net number of information bits that are transmitted without error per unit time (sometimes referred to as goodput). It can be expressed as

$$
T_{k}=\frac{L}{M} R_{k} f\left(\gamma_{k}\right)
$$

where $L$ and $M$ are the number of information bits and the total number of bits in a packet, respectively; $R_{k}$ and $\gamma_{k}$ are the transmission rate and the SINR for the $k$ th user, respectively; and $f\left(\gamma_{k}\right)$ is the efficiency function representing the packet success rate (PSR), i.e., the probability that a packet is received without an error. Our assumption is that if a packet has one or more bit errors, it will be retransmitted. The efficiency function, $f(\cdot)$, is assumed to be increasing, continuous, and S-shaped ${ }^{1}$ (sigmoidal)

\footnotetext{
${ }^{1}$ An increasing function is S-shaped if there is a point above which the function is concave, and below which the function is convex.
} 


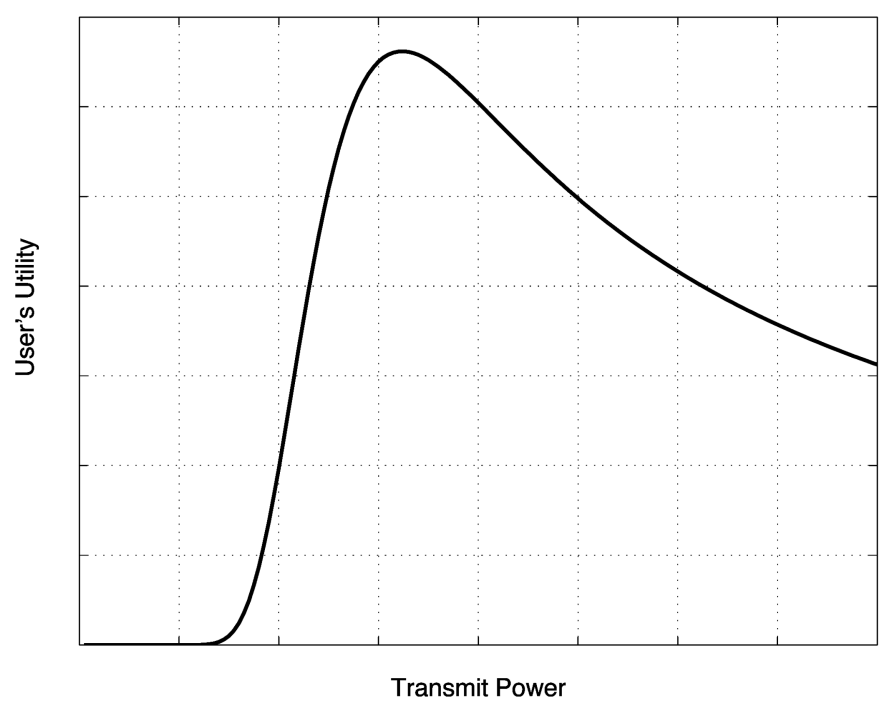

Fig. 2. User's utility as a function of transmit power for fixed interference (single-carrier case).

with $f(\infty)=1$. We also require that $f(0)=0$ to ensure that $u_{k}=0$ when $p_{k}=0$. These assumptions are valid in many practical systems. An example of a sigmoidal efficiency function is given in Fig. 1. Using a sigmoidal efficiency function, the shape of the utility function in (1) is shown in Fig. 2 as a function of the user's transmit power keeping other users' transmit powers fixed. It should be noted that the throughput $T_{k}$ in (2) could be replaced with any increasing concave function as long as we make sure that $u_{k}=0$ when $p_{k}=0$. A more detailed discussion of the efficiency function can be found in [9]. It can be shown that for a sigmoidal efficiency function, the utility function in (1) is a quasi-concave ${ }^{2}$ function of the user's transmit power [17]. This is also true if the throughput in (2) is replaced with an increasing concave function of $\gamma_{k}$.

Based on (1) and (2), the utility function for user $k$ can be written as

$$
u_{k}=\left(\frac{L}{M} R_{k}\right) \frac{f\left(\gamma_{k}\right)}{p_{k}}
$$

This utility function, which has units of bits/joule, captures very well the tradeoff between throughput and battery life and is particularly suitable for applications where energy efficiency is crucial.

Power control is modeled as a noncooperative game in which each user tries to selfishly maximize its own utility. It is shown in [4] that, in a single-carrier system, when MFs are used as the uplink receivers, if user terminals are allowed to choose only their transmit powers for maximizing their utilities, then there exists an equilibrium point at which no user can improve its utility given the power levels of other users (Nash equilibrium). This equilibrium is achieved when the users' transmit powers are SINR-balanced with the output SINR being equal to $\gamma^{*}$, the solution to $f(\gamma)=\gamma f^{\prime}(\gamma)$. Furthermore, this equilibrium is unique. In [9], this analysis is extended to other linear receivers.

\footnotetext{
${ }^{2}$ The function $f$ defined on a convex set $\mathcal{S}$ is quasi-concave if every superlevel set of $f$ is convex, i.e., $\{x \in \mathcal{S} \mid f(x) \geq a\}$ is convex for every value of $a$.
}

In this work, we extend this game-theoretic approach to multicarrier systems. In the multicarrier case, each user's strategy is a vector (rather than a scalar). Furthermore, the utility function is not a quasi-concave function of the user's strategy. Hence, the problem is much more challenging than the one in the singlecarrier scenario.

\section{NONCOOPERATIVE POWER CONTROL GAME IN MULTICARRIER SYSTEMS}

Let us consider the uplink of a synchronous multicarrier DS-CDMA data network with $K$ users, $D$ carriers and processing gain $N$ (for each carrier). The carriers are assumed to be sufficiently far apart so that the (spread-spectrum) signal transmitted over each carrier does not interfere with the signals transmitted over other carriers [11]. We also assume that the delay spread and Doppler spread are negligible for each individual carrier. At the transmitter, the incoming bits for user $k$ are divided into $D$ parallel streams and each stream is spread using the spreading code of user $k$. The $D$ parallel streams are then sent over the $D$ (orthogonal) carriers. For the $\ell$ th carrier, the received signal at the uplink receiver (after chip-matched filtering and sampling) can be represented by an $N \times 1$ vector as

$$
\mathbf{r}_{\ell}=\sum_{k=1}^{K} \sqrt{p_{k \ell} h_{k \ell}} b_{k \ell} \mathbf{s}_{k}+\mathbf{w}_{\ell}
$$

where $b_{k \ell}, p_{k \ell}, h_{k \ell}$ are the $k$ th user's transmitted bit, transmit power and path gain, respectively, for the $\ell$ th frequency channel (carrier); $\mathbf{s}_{k}$ is the spreading sequence for user $k$ which is assumed to be random with unit norm; and $\mathbf{w}_{\ell}$ is the noise vector which is assumed to be Gaussian with mean $\mathbf{0}$ and covariance $\sigma^{2} \mathbf{I}$. Let us express the channel gain as

$$
h_{k \ell}=\frac{c}{d_{k}^{\nu}} a_{k \ell}^{2}
$$

where $d_{k}$ is the distance of user $k$ from the uplink receiver and $a_{k \ell}$ is a Rayleigh random variable representing the small scale channel fading. Here, $c$ and $\nu$ are constants which determine the path loss as a function of distance.

We propose a noncooperative game in which each user chooses its transmit powers over the $D$ carriers to maximize its overall utility. In other words, each user (selfishly) decides how much power to transmit over each frequency channel (carrier) to achieve the highest overall utility. Let $G_{D}=\left[\mathcal{K},\left\{A_{k}^{\mathrm{MC}}\right\},\left\{u_{k}^{\mathrm{MC}}\right\}\right]$ denote the proposed noncooperative game where $\mathcal{K}=\{1, \ldots, K\}$, and $A_{k}^{\mathrm{MC}}=\left[0, P_{\max }\right]^{D}$ is the strategy set for the $k$ th user. Here, $P_{\max }$ is the maximum transmit power on each carrier. Each strategy in $A_{k}^{\mathrm{MC}}$ can be written as $\mathbf{p}_{k}=\left[p_{k 1}, \ldots, p_{k D}\right]$. The utility function for user $k$ is defined as the ratio of the total throughput to the total transmit power for the $D$ carriers, i.e.,

$$
u_{k}^{\mathrm{MC}}=\frac{\sum_{\ell=1}^{D} T_{k \ell}}{\sum_{\ell=1}^{D} p_{k \ell}}
$$

where $T_{k \ell}$ is the throughput achieved by user $k$ over the $\ell$ th carrier, and is given by $T_{k \ell}=(L / M) R_{k} f\left(\gamma_{k \ell}\right)$ with $\gamma_{k \ell}$ denoting 
the received SINR for user $k$ on carrier $\ell$. Hence, the resulting noncooperative game can be expressed as the following maximization problem:

$$
\max _{\mathbf{p}_{k}} u_{k}^{\mathrm{MC}}=\max _{p_{k 1}, \ldots, p_{k D}} u_{k}^{\mathrm{MC}} \quad \text { for } k=1, \ldots, K
$$

under the constraint of nonnegative powers (i.e., $p_{k \ell} \geq 0$ for all $k=1, \ldots, K$ and $\ell=1, \ldots, D$ ). Without significant loss of generality, if we assume equal transmission rates for all users, (7) can be expressed as

$$
\max _{p_{k 1}, \ldots, p_{k D}} \frac{\sum_{\ell=1}^{D} f\left(\gamma_{k \ell}\right)}{\sum_{\ell=1}^{D} p_{k \ell}} \quad \text { for } k=1, \ldots, K .
$$

The relationship between the $\gamma_{k \ell}$ 's and the $p_{k \ell}$ 's is dependent on the uplink receiver.

It should be noted that the assumption of equal transmission rates for all users can be made less restrictive. For our analysis, it is sufficient for the users to have equal transmission rates over different carriers but the transmission rate can be different for different users. More generally, the proposed power control game can be extended to allow the users to pick not only their transmit powers but also their transmission rates over the $D$ carriers. While joint power and rate control is important, particularly for data applications, our focus throughout this work is on power control only (see [21] for a recent result on joint optimization of power and rate in single-carrier case). We will briefly comment on the joint power and rate control problem at the end of Section IV.

\section{NASh EQUilibrium For THE PROPOSED GAME}

For the noncooperative power control game proposed in the previous section, a Nash equilibrium is a set of power vectors, $\mathbf{p}_{1}^{*}, \ldots, \mathbf{p}_{K}^{*}$, such that no user can unilaterally improve its utility by choosing a different power vector, i.e., $\mathbf{p}_{1}^{*}, \ldots, \mathbf{p}_{K}^{*}$ is a Nash equilibrium if and only if

$$
u_{k}^{\mathrm{MC}}\left(\mathbf{p}_{k}^{*}, \mathbf{P}_{-k}^{*}\right) \geq u_{k}^{\mathrm{MC}}\left(\mathbf{p}_{k}, \mathbf{P}_{-k}^{*}\right) \text { for all } \mathbf{p}_{k}
$$

and for $k=1, \ldots, K$. Here, $\mathbf{P}_{-k}^{*}$ denotes the set of transmit power vectors of all the users except for user $k$.

We begin by characterizing utility maximization by a singleuser when other users' transmit powers are fixed.

Proposition 1: For all linear receivers and with all other users' transmit powers being fixed, user $k$ 's utility function, given by (6), is maximized when

$$
p_{k \ell}= \begin{cases}p_{k L_{k}}^{*}, & \text { for } \ell=L_{k} \\ 0, & \text { for } \ell \neq L_{k}\end{cases}
$$

where $L_{k}=\arg \min _{\ell} p_{k \ell}^{*}$ with $p_{k \ell}^{*}$ being the transmit power required by user $k$ to achieve an output SINR equal to $\gamma^{*}$ on the $\ell$ th carrier, or $P_{\max }$ if $\gamma^{*}$ cannot be achieved. Here, $\gamma^{*}$ is the unique (positive) solution of $f(\gamma)=\gamma f^{\prime}(\gamma)$.

Proof: We first show that $f(\gamma) / p$ is maximized when $p$ is such that $\gamma=\gamma^{*}$. For this, we take the derivative of $f(\gamma) / p$ with respect to $p$ and equate it to zero to obtain

$$
p \frac{\partial \gamma}{\partial p} f^{\prime}(\gamma)-f(\gamma)=0
$$

Since for all linear receivers $p(\partial \gamma / \partial p)=\gamma[9], f(\gamma) / p$ is maximized when $\gamma=\gamma^{*}$, the (positive) solution to $f(\gamma)=\gamma f^{\prime}(\gamma)$. It is shown in [17] that for an S-shaped function, $\gamma^{*}$ exists and is unique. If $\gamma^{*}$ cannot be achieved, $f(\gamma) / p$ is maximized when $p=P_{\max }$. Now, define $p_{k \ell}^{*}$ as the transmit power required by user $k$ to achieve an output SINR equal to $\gamma^{*}$ on the $\ell$ th carrier (or $P_{\max }$ if $\gamma^{*}$ is not achievable) and let $L_{k}=\arg \min _{\ell} p_{k \ell}^{*}$. In case of ties, we can pick any of the indices corresponding to the minimum power. Then, based on the above argument, we have $f\left(\gamma_{k L_{k}}\right) / p_{k L_{k}} \leq f\left(\gamma^{*}\right) / p_{k L_{k}}^{*}$ for any $p_{k L_{k}} \geq 0$. Also, because $p_{k L_{k}}^{*}=\min _{\ell} p_{k \ell}^{*}$, we have $f\left(\gamma_{k \ell}\right) / p_{k \ell} \leq f\left(\gamma^{*}\right) / p_{k \ell}^{*} \leq$ $f\left(\gamma^{*}\right) / p_{k L_{k}}^{*}$ for all $p_{k \ell} \geq 0$ and $\ell \neq L_{k}$. Based on the above inequalities, we can write

$$
\frac{f\left(\gamma_{k \ell}\right)}{f\left(\gamma^{*}\right)} \leq \frac{p_{k \ell}}{p_{k L_{k}}^{*}}, \quad \text { for } \ell=1,2, \ldots, D .
$$

Adding the $D$ inequalities given in (12) and rewriting the resulting inequality, we have

$$
\frac{\sum_{\ell=1}^{D} f\left(\gamma_{k \ell}\right)}{\sum_{\ell=1}^{D} p_{k \ell}} \leq \frac{f\left(\gamma^{*}\right)}{p_{k L_{k}}^{*}} \text { for all } p_{k 1}, \ldots, p_{k D} \geq 0
$$

This completes the proof.

Proposition 1 suggests that the utility for user $k$ is maximized when the user transmits only over its "best" carrier such that the achieved SINR at the output of the uplink receiver is equal to $\gamma^{*}$. The "best" carrier is the one that requires the least amount of transmit power to achieve $\gamma^{*}$ at the output of the uplink receiver. Based on Proposition 1, at a Nash equilibrium each user transmits only on one carrier. This significantly reduces the number of cases that need to be considered as possible candidates for a Nash equilibrium. A set of power vectors, $\mathbf{p}_{1}^{*}, \ldots, \mathbf{p}_{K}^{*}$, is a Nash equilibrium if and only if they simultaneously satisfy (10).

It should also be noted that the utility-maximizing strategy suggested by Proposition 1 is different from the waterfilling approach that is discussed in [18] for digital subscriber line (DSL). This is because in [18], utility is defined as the user's throughput and the goal there is to maximize this utility function for a fixed amount of available power. Here, on the other hand, the amount of available power is not fixed. In addition, utility is defined here as the number of bits transmitted per joule of energy consumed which is particularly suitable for wireless systems with energy constraints.

Alternatively, user $k$ 's utility function can be defined as $\tilde{u}_{k}=$ $\sum_{\ell=1}^{D} T_{k \ell} / p_{k \ell}$. This utility function is maximized when each of the terms in the summation is maximized. This happens when the user transmits on all the carriers at power levels that achieve $\gamma^{*}$ for every carrier. This is equivalent to the case in which each user maximizes its utility over each carrier independently. We show in Section VIII that our proposed joint maximization approach, through performing a distributed interference avoidance mechanism, significantly outperforms the approach of individual utility maximization. Throughout this paper, the expression in (6) is used for the user's utility function.

Since at Nash equilibrium (when it exists), each user must transmit on one carrier only, there are exactly $D^{K}$ possibilities 
for an equilibrium. For example, in the case of $K=D=2$, there are four possibilities for Nash equilibrium.

- User 1 and user 2 both transmit on the first carrier.

- User 1 and user 2 both transmit on the second carrier.

- User 1 transmits on the first carrier and user 2 transmits on the second carrier.

- User 1 transmits on the second carrier and user 2 transmits on the first carrier.

Depending on the set of channel gains, i.e., the $h_{k \ell}$ 's, the proposed power control game may have no equilibrium, a unique equilibrium, or more than one equilibrium. In the following, we investigate the existence and uniqueness of Nash equilibrium for the conventional MF receiver and also comment on the extensions of the results to other linear multiuser receivers such as the decorrelating and minimum-mean-square-error (MMSE) detectors [19], [20].

For the joint power and rate control problem, it can be shown by using a similar technique as the one used in the proof of Proposition 1 that for each user to maximize its own utility, the user must transmit only on its "best" carrier. Furthermore, the combined choice of power and rate has to be such that the output SINR is equal to $\gamma^{*}$. This implies that there are infinite combinations of power and rate that maximize the user's utility given that the powers and rates of other users are fixed.

\section{EXISTENCE AND UNIQUENESS OF NASH EQUILIBRIUM}

If we assume random spreading sequences, the output SINR for the $\ell$ th carrier of the $k$ th user with a MF receiver is given by

$$
\gamma_{k \ell}=\frac{p_{k \ell} h_{k \ell}}{\sigma^{2}+\frac{1}{N} \sum_{j \neq k} p_{j \ell} h_{j \ell}} .
$$

Let us define

$$
\hat{h}_{k \ell}=\frac{h_{k \ell}}{\sigma^{2}+\frac{1}{N} \sum_{j \neq k} p_{j \ell} h_{j \ell}}
$$

as the "effective channel gain" for user $k$ over the $\ell$ th carrier. Based on (14) and (15), we have $\gamma_{k \ell}=\hat{h}_{k \ell} p_{k \ell}$.

Let us for now assume that the processing gain $N$ is sufficiently large so that even when all $K$ users transmit on the same carrier, $\gamma^{*}$ can be achieved by all users. This is the case when $N>(K-1) \gamma^{*}$. We later relax this assumption. The following proposition helps identify the Nash equilibrium (when it exists) for a given set of channel gains.

Proposition 2: For a MF receiver, a necessary condition for user $k$ to transmit on the $\ell$ th carrier at equilibrium is that

$$
\frac{h_{k \ell}}{h_{k i}}>\frac{\Theta_{n(\ell)}}{\Theta_{n(i)}} \Theta_{0} \quad \text { for all } i \neq \ell
$$

where $n(i)$ is the number of users transmitting on the $i$ th carrier and

$$
\Theta_{n}=\frac{1}{1-(n-1) \frac{\gamma^{*}}{N}} \quad n=0,1, \ldots, K .
$$

In this case, $p_{k \ell}^{*}=\left(\gamma^{*} \sigma^{2} / h_{k \ell}\right) \Theta_{n(\ell)}$.
Proof: Based on Proposition 1, in order for user $k$ to transmit on carrier $\ell$ at equilibrium, we must have

$$
\hat{h}_{k \ell}>\hat{h}_{k i} \text { for all } i \neq \ell \text {. }
$$

Since $n(\ell)$ users (including user $k$ ) are transmitting on the $\ell$ th carrier and $n(i)$ users are transmitting on the $i$ th carrier and all users have an output SINR equal to $\gamma^{*}$, we have

$$
\hat{h}_{k \ell}=\frac{h_{k \ell}}{\sigma^{2}+\frac{n(\ell)-1}{N} q \ell}
$$

and

$$
\hat{h}_{k i}=\frac{h_{k i}}{\sigma^{2}+\frac{n(i)}{N} q_{i}}
$$

where

$$
q_{\ell}=\sigma^{2} \gamma^{*}\left[1-\frac{(n(\ell)-1) \gamma^{*}}{N}\right]^{-1}
$$

and

$$
q_{i}=\sigma^{2} \gamma^{*}\left[1-\frac{(n(i)-1) \gamma^{*}}{N}\right]^{-1}
$$

are the received powers for each user on the $\ell$ th and $i$ th carriers, respectively. Now, define

$$
\Theta_{n}=\left[1-\frac{(n-1) \gamma^{*}}{N}\right]^{-1}
$$

to get $q_{\ell}=\sigma^{2} \gamma^{*} \Theta_{n(\ell)}$ and $q_{i}=\sigma^{2} \gamma^{*} \Theta_{n(i)}$. Substituting $q_{\ell}$ and $q_{i}$ into (19) and (20) and taking advantage of the fact that $1+\left((n-1) \gamma^{*} / N\right) \Theta_{n}=\Theta_{n}$, we get

$$
\hat{h}_{k \ell}=\frac{h_{k \ell}}{\Theta_{n(\ell)} \sigma^{2}}
$$

and

$$
\hat{h}_{k i}=\frac{h_{k i}}{\frac{\Theta_{n(i)}}{\Theta_{0}} \sigma^{2}} .
$$

Consequently, (16) is obtained by substituting (21) and (22) into (18). Furthermore, since $p_{k \ell}^{*} h_{k \ell}=q_{\ell}=\sigma^{2} \gamma^{*} \Theta_{n(\ell)}$, we have $p_{k \ell}^{*}=\left(\gamma^{*} \sigma^{2} / h_{k \ell}\right) \Theta_{n(\ell)}$, and this completes the proof.

Note that, based on (17), when $N>(K-1) \gamma^{*}$, we have $0<\Theta_{0}<\Theta_{1}<\Theta_{2}<\cdots<\Theta_{K}$ with $\Theta_{1}=1$.

For each of the $D^{K}$ possible equilibria, the channel gains for each user must satisfy $D-1$ inequalities similar to (16). Furthermore, satisfying a set of $K(D-1)$ of such inequalities by the $K$ users is sufficient for existence of Nash equilibrium but the uniqueness is not guaranteed. For example, for the case of $K=D=2$, the four possible equilibria can be characterized as follows.

- For both users to transmit on the first carrier at equilibrium, we must have $h_{11} / h_{12}>\Theta_{2}$ and $h_{21} / h_{22}>\Theta_{2}$.

- For both users to transmit on the second carrier at equilibrium, we must have $h_{11} / h_{12}<1 / \Theta_{2}$ and $h_{21} / h_{22}<$ $1 / \Theta_{2}$. 


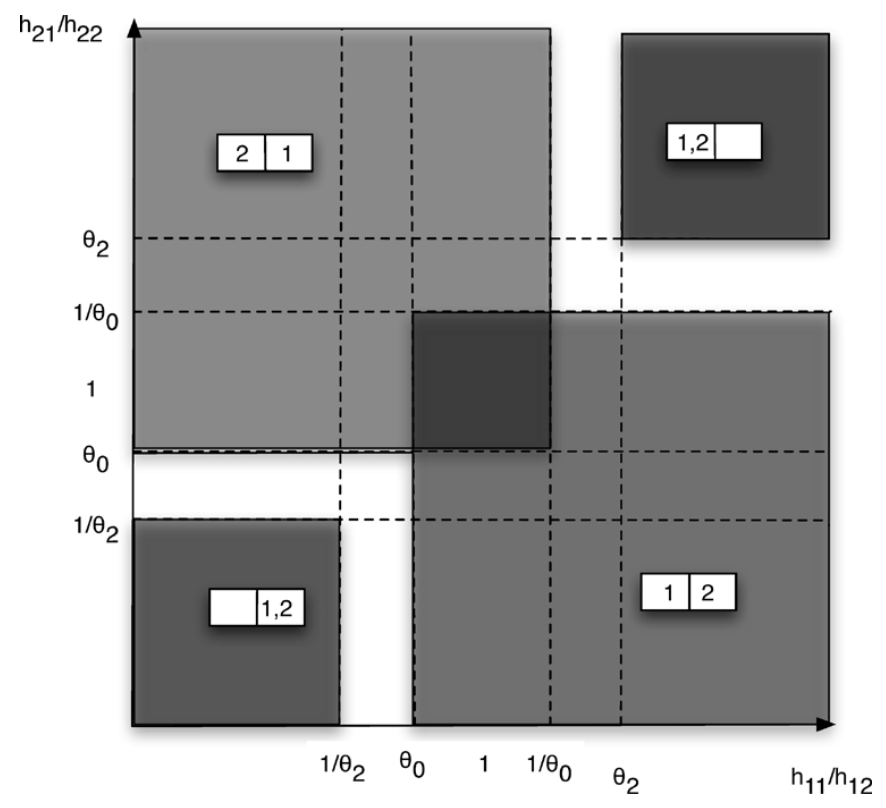

Fig. 3. Nash equilibrium regions for the case of two users and two carriers. (1, 2), for example, means that at equilibrium user 1 transmits on the first carrier and user 2 transmits on the second carrier.

- For user 1 and user 2 to transmit on the first and second carriers, respectively, at equilibrium, we must have $h_{11} / h_{12}>\Theta_{0}$ and $h_{21} / h_{22}<1 / \Theta_{0}$.

- For user 1 and user 2 to transmit on the second and first carriers, respectively, at equilibrium, we must have $h_{11} / h_{12}<1 / \Theta_{0}$ and $h_{21} / h_{22}>\Theta_{0}$.

Fig. 3 shows the regions corresponding to the above four equilibria. It can be seen that for certain values of channel gains there is no Nash equilibrium (the white areas in the figure) and for some values of channel gains there are two possible equilibria. When the channel gains belong to the white regions, none of the four possible candidates for Nash equilibrium is stable. It can be shown that if we put the two users in any of these four possible states, at least one of the users would prefer to jump to the other carrier because that would improve its utility. Hence, no Nash equilibrium exists.

It is interesting to observe that homogeneity of channel gains can prevent Nash equilibrium from existing. Consider the twouser two-carrier case where $h_{12}=h_{21}$, it is easy to verify the following.

Corollary 1: If either $h_{11} / h_{22}$ or $h_{22} / h_{11}$ belongs to $\left[1 / \Theta_{2}^{2}, \Theta_{0}^{2}\right]$, then there does not exist a Nash equilibrium.

When the processing gain is less than or equal to $(K-1) \gamma^{*}$, it is not possible for all users to transmit on the same carrier and achieve $\gamma^{*}$ simultaneously. Instead, they would end up transmitting at the maximum power (see Proposition 1). More specifically, for any given $N \leq(K-1) \gamma^{*}$, at most $\left\lfloor N / \gamma^{*}-1\right\rfloor$ users can simultaneously achieve $\gamma^{*}$ on the same carrier. Therefore, as $N$ decreases, those Nash equilibria in which the distribution of users among the carriers is less uniform are less likely to occur. This means that for small values of processing gain, the distribution of users among the carriers becomes more uniform. However, the probability that no Nash equilibrium exists increases. We will discuss this further in Section VI.
Similar analysis can be done for the decorrelating and MMSE detectors. However, because of the dependence of the SINR expressions of these receivers on the spreading sequences of all the users, obtaining closed form expressions such as the ones given in (16) and (17) has not been possible.

\section{Special CaSe of Two Carriers}

To gain some insight into the properties of the Nash equilibria for our proposed game, let us concentrate on a system with two carriers and two users (i.e., $D=K=2$ ). We assume that the $a_{k \ell}$ 's in (5) are independent and identically distributed (i.i.d.) among the users and carriers and have the Rayleigh distribution with mean 1. As a result, the $a_{k \ell}^{2}$ 's will be i.i.d. with the exponential distribution of mean 1 . Let $X_{1}$ be the random variable corresponding to the number of users that transmit over the first carrier at equilibrium. Also, let $P_{X_{1}}(m)$ represent the probability that there are $m$ users on the first carrier at Nash equilibrium.

Proposition 3: If $N>\gamma^{*}$, then the probabilities that there are zero, one, and two users transmitting on the first carrier at Nash equilibrium are, respectively, given by

$$
\begin{aligned}
& P_{X_{1}}(0)=\left(\frac{1}{1+\Theta_{2}}\right)^{2} \\
& P_{X_{1}}(1)=2\left(\frac{1}{1+\Theta_{0}}\right)^{2}-\left(\frac{1-\Theta_{0}}{1+\Theta_{0}}\right)^{2} \\
& P_{X_{1}}(2)=\left(\frac{1}{1+\Theta_{2}}\right)^{2} .
\end{aligned}
$$

Proof: If we assume $N>\gamma^{*}$, then, based on Proposition 2 , the probability that both users transmit on the first carrier at equilibrium (i.e., $X_{1}=2$ ) is given by (see Fig. 3 )

$$
\begin{aligned}
P_{X_{1}}(2) & =\operatorname{Pr}\left\{X_{1}=2\right\}=\operatorname{Pr}\left\{\frac{h_{11}}{h_{12}}>\Theta_{2}, \frac{h_{21}}{h_{22}}>\Theta_{2}\right\} \\
& =\operatorname{Pr}\left\{\frac{a_{11}^{2}}{a_{12}^{2}}>\Theta_{2}\right\} \operatorname{Pr}\left\{\frac{a_{21}^{2}}{a_{22}^{2}}>\Theta_{2}\right\} \\
& =\left(\iint_{\left\{(u, v): u \geq 0, v \geq 0 \text { and } u / v>\Theta_{2}\right\}} e^{-(u+v)} d u d v\right)^{2} \\
& =\left(\frac{1}{1+\Theta_{2}}\right)^{2} .
\end{aligned}
$$

Similarly, the probability of both users transmitting on the second carrier at equilibrium is

$$
\begin{aligned}
P_{X_{1}}(0) & =\operatorname{Pr}\left\{X_{1}=0\right\} \\
& =\operatorname{Pr}\left\{\frac{h_{12}}{h_{11}}>\Theta_{2}, \frac{h_{22}}{h_{21}}>\Theta_{2}\right\}=\left(\frac{1}{1+\Theta_{2}}\right)^{2} .
\end{aligned}
$$


The probability of one user transmitting on each of the two carriers can be found to be

$$
\begin{aligned}
P_{X_{1}}(1)= & \operatorname{Pr}\left\{X_{1}=1\right\}=\operatorname{Pr}\left\{\frac{h_{11}}{h_{12}}>\Theta_{0}, \frac{h_{21}}{h_{22}}<\frac{1}{\Theta_{0}}\right\} \\
& +\operatorname{Pr}\left\{\frac{h_{11}}{h_{12}}<\frac{1}{\Theta_{0}}, \frac{h_{21}}{h_{22}}>\Theta_{0}\right\} \\
& -\operatorname{Pr}\left\{\Theta_{0}<\frac{h_{11}}{h_{12}}<\frac{1}{\Theta_{0}}, \Theta_{0}<\frac{h_{21}}{h_{22}}<\frac{1}{\Theta_{0}}\right\} \\
= & 2\left(\frac{1}{1+\Theta_{0}}\right)^{2}-\left(\frac{1-\Theta_{0}}{1+\Theta_{0}}\right)^{2} .
\end{aligned}
$$

This completes the proof.

Based on (23)-(25), the probability that no Nash equilibrium exists is given by

$$
\begin{aligned}
P_{o} & =\operatorname{Pr}\{\text { No Nash equilibrium }\} \\
& =2\left\{\left(\frac{\Theta_{0}}{1+\Theta_{0}}\right)^{2}-\left(\frac{1}{1+\Theta_{2}}\right)^{2}\right\} .
\end{aligned}
$$

It should be noted that since $P_{o}>0$, the $P_{X_{1}}(m)$ 's, in general, do not add up to 1 . Therefore, they represent a pseudo probability mass function (pseudo PMF) for $X_{1}$. As the processing gain $N$ becomes larger, $\Theta_{0}$ and $\Theta_{2}$ approach 1 from below and above, respectively. This results in a reduction in $P_{X_{1}}(1)$ but an increase in $P_{X_{1}}(0)$ and $P_{X_{1}}(2)$, i.e., the pseudo PMF for $X_{1}$ becomes flatter. However, the increase outweighs the decrease and as a result $P_{o}$ decreases as $N$ increases. Going back to Fig. 3, we see that the region for which no Nash equilibrium exists shrinks as $N$ increases. In addition, the region for which more than one equilibrium exists disappears as $N$ becomes very large. Therefore, as the processing gain becomes large, the probability that the proposed power control game has a unique Nash equilibrium approaches one. Note that as $N \rightarrow \infty$, we have $\Theta_{0} \rightarrow 1$ and $\Theta_{2} \rightarrow 1$. Therefore, based on (23)-(27), in the limit of large processing gains, we have $P_{X_{1}}(0)=P_{X_{1}}(2)=1 / 4$, $P_{X_{1}}(1)=1 / 2$ and $P_{o}=0$. This is because for a sufficiently large processing gain, the multiple-access interference becomes insignificant compared with the background noise. Therefore, each user transmits on the carrier with the best channel gain. This means that the probability that user 1 transmits on the first carrier becomes independent of whether the other user is transmitting on the first or second carrier (and vice versa).

So far, the assumption has been that $N>\gamma^{*}$ so that both users can achieve $\gamma^{*}$ even when they are transmitting over the same carrier.

Proposition 4: If $N \leq \gamma^{*}$, then the probabilities that there are zero, one, and two users transmitting on the first carrier at Nash equilibrium are, respectively, given by

$$
\begin{aligned}
& P_{X_{1}}(0) \approx 0 \\
& P_{X_{1}}(1)=2\left(\frac{1}{1+\Theta_{0}}\right)^{2}-\left(\frac{1-\Theta_{0}}{1+\Theta_{0}}\right)^{2} \\
& P_{X_{1}}(2) \approx 0 .
\end{aligned}
$$

Proof: If $N \leq \gamma^{*}$, the users cannot achieve $\gamma^{*}$ simultaneously when they are transmitting on the same carrier and they would end up transmitting at the maximum power. Hence

$$
P_{X_{1}}(2)=\operatorname{Pr}\left\{X_{1}=2\right\}=\operatorname{Pr}\left\{P_{\max }<p_{12}^{*} \text { and } P_{\max }<p_{22}^{*}\right\}
$$

where $p_{12}^{*}=\sigma^{2} \gamma^{*} / h_{12}$ and $p_{22}^{*}=\sigma^{2} \gamma^{*} / h_{22}$. Therefore

$$
\begin{aligned}
P_{X_{1}}(2) & =\operatorname{Pr}\left\{h_{12}<\frac{\sigma^{2} \gamma^{*}}{P_{\max }} \text { and } h_{22}<\frac{\sigma^{2} \gamma^{*}}{P_{\max }}\right\} \\
& =\operatorname{Pr}\left\{h_{12}<\frac{\sigma^{2} \gamma^{*}}{P_{\max }}\right\} \operatorname{Pr}\left\{h_{122}<\frac{\sigma^{2} \gamma^{*}}{P_{\max }}\right\}
\end{aligned}
$$

assuming independent channel gains.

Now, based on (5), we have

$$
P_{X_{1}}(2)=\operatorname{Pr}\left\{a_{12}^{2}<\frac{d_{1}^{\nu} \sigma^{2} \gamma^{*}}{c P_{\max }}\right\} \operatorname{Pr}\left\{a_{22}^{2}<\frac{d_{2}^{\nu} \sigma^{2} \gamma^{*}}{c P_{\max }}\right\} .
$$

If the channel amplitudes have a Rayleigh distribution, then $a_{k \ell}^{2}$ 's have an exponential distribution with mean 1 . Therefore

$$
\operatorname{Pr}\left\{a_{k \ell}^{2}<\frac{d_{k}^{\nu} \sigma^{2} \gamma^{*}}{c P_{\max }}\right\}=1-e^{-b_{k \ell}}
$$

where

$$
b_{k \ell}=\frac{d_{k}^{\nu} \sigma^{2} \gamma^{*}}{c P_{\max }}
$$

For typical values of $c, d, \nu, \sigma^{2}$, and $P_{\max }, b$ is very small. For example, for $c=0.1, d=100 \mathrm{~m}, \nu=4, \sigma^{2}=10^{-16} \mathrm{~W}$, $\gamma^{*}=6.4$, and $P_{\max }=1 \mathrm{~W}$, we get $b=6.4 \times 10^{-7}$. As a result, $1-e^{-b} \simeq b$. Therefore, $P_{X_{1}}(2) \cong b_{12} b_{22} \approx 0$. Similarly, we have $P_{X_{1}}(0) \cong b_{11} b_{21} \approx 0$.

To obtain $P_{X_{1}}(1)$, we can follow the same steps as the ones used for the case of $N>\gamma^{*}$. Hence, when $N \leq \gamma^{*}$, we have $P_{X_{1}}(1)=2\left(1 /\left(1+\Theta_{0}\right)\right)^{2}-\left(\left(1-\Theta_{0}\right) /\left(1+\Theta_{0}\right)\right)^{2}$.

This complete the proof.

Based on (27)-(29), we have

$$
P_{o}=1-\left[2\left(\frac{1}{1+\Theta_{0}}\right)^{2}-\left(\frac{1-\Theta_{0}}{1+\Theta_{0}}\right)^{2}\right]=2\left(\frac{\Theta_{0}}{1+\Theta_{0}}\right)^{2}
$$

when $N \leq \gamma^{*}$.

We can summarize Propositions 3 and 4 as

$$
\begin{aligned}
& P_{X_{1}}(0)=P_{X_{1}}(2)= \begin{cases}0, & \text { if } N \leq \gamma^{*} \\
\left(\frac{1}{1+\Theta_{2}}\right)^{2}, & \text { if } N>\gamma^{*}\end{cases} \\
& P_{X_{1}}(1)=2\left(\frac{1}{1+\Theta_{0}}\right)^{2}-\left(\frac{1-\Theta_{0}}{1+\Theta_{0}}\right)^{2}
\end{aligned}
$$

and

$$
P_{o}=\left\{\begin{array}{ll}
2\left(\frac{\Theta_{0}}{1+\Theta_{0}}\right)^{2}, & \text { if } N \leq \gamma^{*} \\
2\left[\left(\frac{\Theta_{0}}{1+\Theta_{0}}\right)^{2}-\left(\frac{1}{1+\Theta_{2}}\right)^{2}\right], & \text { if } N>\gamma^{*}
\end{array} .\right.
$$


Although obtaining explicit expressions for the probabilities of the occurrence of various Nash equilibria for the case in which $K>2$ is more complicated, many of the general trends observed for the $K=2$ case are also valid when $K>2$. Namely, as $N$ increases the pseudo PMF of $X_{1}$ becomes wider (i.e., it has larger variance) and at the same time the probability that no equilibrium exists becomes smaller. This means that in the asymptotic case of large processing gains, the proposed power control game has a unique equilibrium. Furthermore, for very large values of $N$, the PMF of $X_{1}$ can be approximated as

$$
\begin{aligned}
P_{X_{1}}(m) & =\operatorname{Pr}\left\{X_{1}=m\right\} \\
& \approx\left(\begin{array}{c}
K \\
m
\end{array}\right)\left(\frac{1}{2}\right)^{K} \text { for } m=0, \ldots, K .
\end{aligned}
$$

Similar arguments can be made for the decorrelating and MMSE detectors. In particular, since these receivers are more powerful in combating interference, for the same processing gain, the pseudo PMF of $X_{1}$ is wider as compared with the one for the MF. Also, since multiuser receivers suppress multiple-access interference more effectively, the probability of presence of a particular user on a certain carrier is almost independent of where the other users are transmitting. This is particularly true for large processing gains. Therefore, the approximation in (33) is more accurate for the decorrelating and MMSE detectors as compared with the conventional MF receiver.

The validity of these claims will also be confirmed through simulations in Section VIII.

\section{A Distributed Power Control Algorithm}

In this section, we present an iterative and distributed algorithm for reaching a Nash equilibrium of the proposed power control game (when it exists). This algorithm is applicable to all linear receivers including the MF, decorrelating and MMSE detectors. The description of the algorithm is as follows.

\section{The Best-Response Multicarrier Power-Control (BMP) Algorithm}

Consider a network with $K$ users and $D$ carriers.

Step 1) Initialize the transmit powers of all the users over all the carriers, and let NumIter $=0$.

Step 2) $\quad$ Set $k=1$, and NumIter $=$ NumIter +1 .

Step 3) Given the transmit powers of other users, user $k$ picks its "best" carrier and transmits only on this carrier at a power level that achieves an output SINR equal to $\gamma^{*}$. The "best" carrier is the one that requires the least amount of transmit power for achieving $\gamma^{*}$.

Step 4) $k=k+1$.

Step 5) If $k \leq K$, then go back to Step 3 .

Step 6) Stop if the powers have converged or if NumIter > MaxNumIter; otherwise go to Step 2.

This is a best-response algorithm since at each stage, a user decides to transmit on the carrier that maximizes the user's utility (i.e., its best-response strategy) given the current conditions of the system. In Step 3, it may appear that each user needs to know not only its own transmit powers and channel gains but also those for all the other users in order to determine its "best" carrier. However, it should be noted that it is actually sufficient for the user to only know its own received SINRs on each carrier. This information can for example be fed back to the user terminal from the access point.

It is clear that if the above algorithm converges, it will converge to a Nash equilibrium. The question that remains to be answered is whether or not the above algorithm converges whenever a Nash equilibrium exists. In Appendix I, we have proved that for the two-user two-carrier case, the BMP algorithm converges to a Nash equilibrium when it exists. Using a similar technique, we have also proved the convergence for the case of two users and $D$ carriers, as well as for the three-user two-carrier case (see Appendices II and III). We have shown that for each of the possible Nash equilibria, starting from any state, the algorithm will eventually reach the equilibrium state. Since the general case of $K$ users and $D$ carriers is not fundamentally different from the ones considered here, the same technique can be used to prove the convergence of the BMP algorithm for the general case of $K$ users and $D$ carriers except that there are many more possibilities to consider in the proof. The convergence of the BMP algorithm has also been demonstrated in Section VIII using extensive simulations. In the case of multiple Nash equilibria, the algorithm converges to one of the equilibria depending on the starting point. For the scenarios where no Nash equilibrium exists, users keep jumping from one carrier to another one when the BMP algorithm is used. However, the algorithm stops when the number of iterations reaches MaxNumIter. The value of MaxNumIter should be chosen large enough to ensure the convergence of the powers for the cases where an equilibrium exists.

\section{Simulation Results}

We first consider the case of two carriers with two users. We assume $L=M=100, R=100 \mathrm{~Kb} / \mathrm{s}$ and $\sigma^{2}=$ $5 \times 10^{-16}$ Watts; and use $f(\gamma)=\left(1-e^{-\gamma}\right)^{M}$ as the efficiency function. ${ }^{3}$ For this efficiency function, $\gamma^{*}=6.4(=8.1 \mathrm{~dB})$. We assume that the channel gains are i.i.d. with exponential distribution of mean 1. We consider 20000 realizations of the channel gains. For each realization, we run the BMP algorithm, proposed in Section VII, for 20 iterations (i.e., MaxNumIter $=20$ ). If convergence is reached by the end of the 20th iteration, we record the number of users that transmit on each carrier; otherwise, we assume there is no equilibrium. For our simulations, $P_{\max }$ is assumed to be very large which translates to having no transmit power limit for the user terminals.

It is observed that the BMP algorithm proposed in Section VII converges whenever a Nash equilibrium exists. Recall that $P_{X_{1}}(m)$ represents the probability that exactly $m$ users transmit on the first carrier at equilibrium. Fig. 4 shows $P_{X_{1}}(2), P_{X_{1}}(1)$, and $P_{O}$ (probability of no equilibrium) as a function of the

\footnotetext{
${ }^{3}$ This is a useful example for the efficiency function and serves as an approximation to the packet success rate that is very reasonable for almost all practical cases with moderate to large values of $M$.
} 


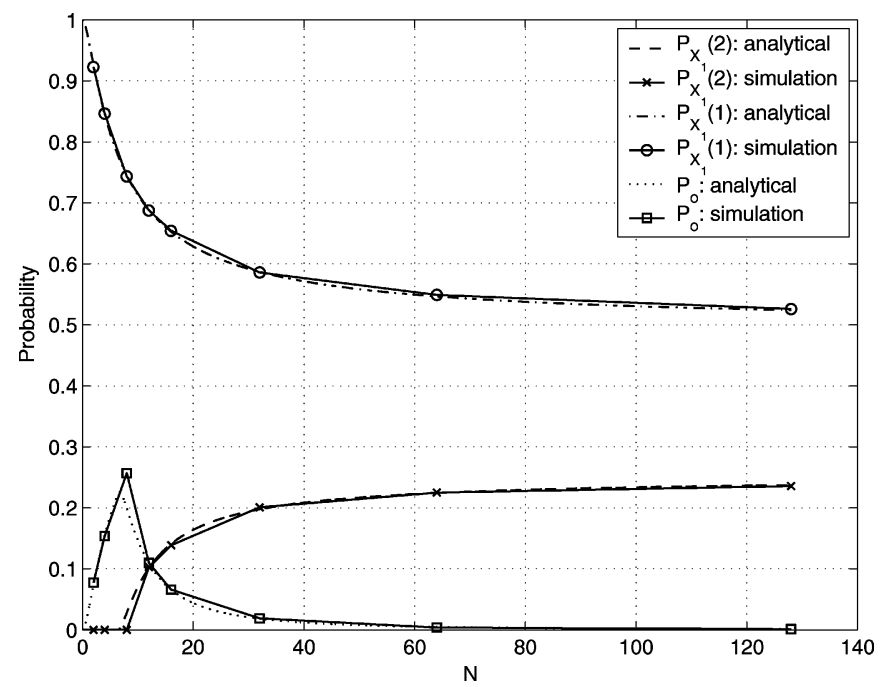

Fig. 4. Probability of having $m$ users on the first carrier at equilibrium $P_{X_{1}}(m)$ for $m=1,2$, as well as probability of having no equilibrium are shown as functions of the processing gain $N$ for the MF for the two-user two-carrier case (i.e., $D=2$ and $K=2$ ).

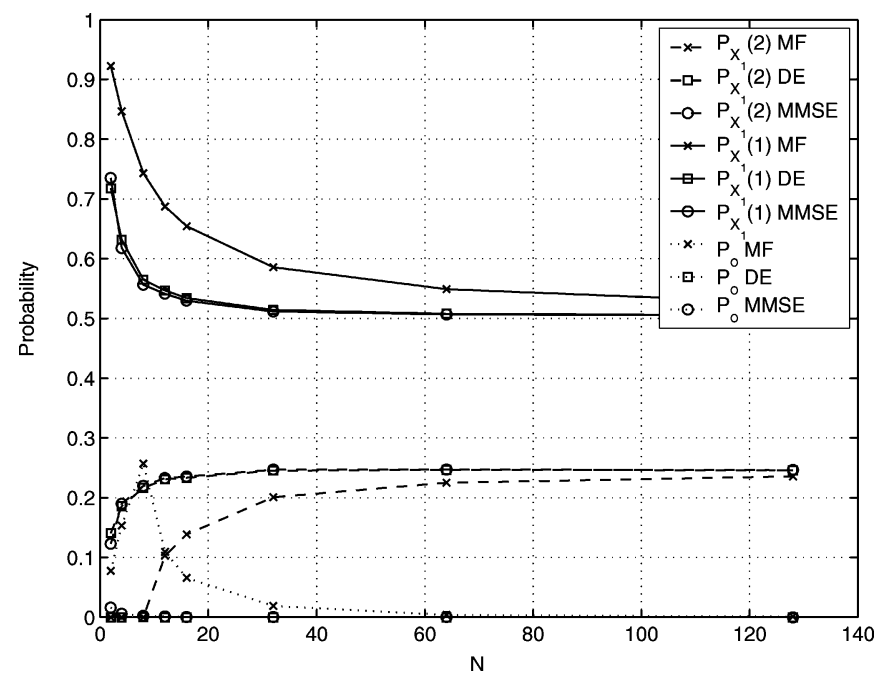

Fig. 5. Probability of having $m$ users on the first carrier at equilibrium $P_{X_{1}}(m)$ for $m=1,2$, as well as probability of having no equilibrium are shown as functions of the processing gain $N$ for the MF, the DE, and the MMSE detector for the two-user two-carrier case (i.e., $D=2$ and $K=2$ ).

processing gain $N$ for the MF. The analytical expressions obtained in Section VI [see (30)-(32)] are also plotted. We see that there is a close agreement between the simulation results and the analytical values. It is also observed that as $N$ becomes large, $P_{o}$ approaches zero. For $N=16$, for example, the probability that a Nash equilibrium exists is about $93 \%$. Since $P_{X_{1}}(0)$ is identical to $P_{X_{1}}(2)$, it is not shown in the figure. For comparison, we have shown the probabilities for the MF, the decorrelator (DE), and the MMSE detector in Fig. 5. It should be noted that $P_{o}$ for the DE is always 0 (as long as $N \geq K$ ) and $P_{o}$ is almost always zero for the MMSE detector (except for low values of $N$ ). This means that for these two receivers a Nash equilibrium almost always exists.

Fig. 6 shows $P_{X_{1}}(m)$ as a function of $m$ for different values of $N$. The results are shown for the MF, the DE, and the MMSE receiver. We can see from the figure that as the processing gain

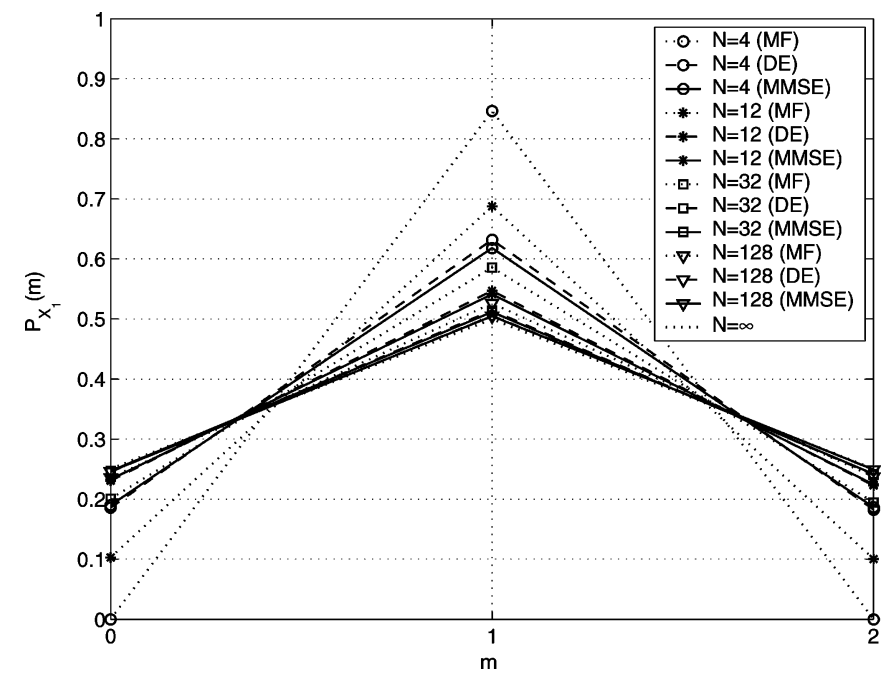

Fig. 6. The (pseudo) probability mass function of $X_{1}$ for different values of processing gain $N$ for the two-user two-carrier case (i.e., $D=2$ and $K=2$ ) for the MF, the DE, and the MMSE detector. $X_{1}$ is a random variable representing the number of users transmitting on the first carrier at Nash equilibrium.

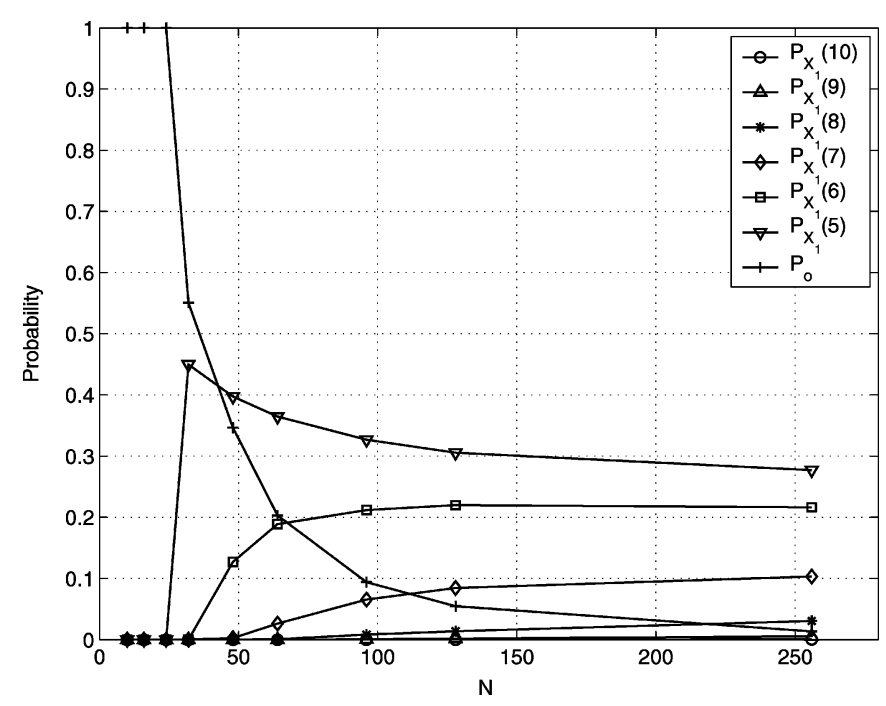

Fig. 7. Probability of having $m$ users on the first carrier at equilibrium $P_{X_{1}}(m)$ for $m=5,6, \ldots, 10$, as well as probability of having no equilibrium are shown as functions of the processing gain $N$ for the MF for the ten-user two-carrier case (i.e., $D=2$ and $K=10$ ).

increases, the pseudo PMF of $X_{1}$ becomes wider (i.e., it has a larger variance). This is because for larger values of $N$, the system becomes more tolerant toward interference. Therefore, the probability with which the two users are able to transmit on the same carrier at equilibrium increases. Since the decorrelating and MMSE detectors are more effective in suppressing interference, for the same processing gain, the corresponding PMFs are wider than that of the MF. We repeat the above experiment for the case of two carriers and ten users. Fig. 7 shows $P_{X_{1}}(m)$ for the MF as a function of the processing gain. Due to symmetry, we have only plotted the probabilities for $m=$ $5,6, \ldots, 10$. The probability that no equilibrium exists (i.e., $P_{o}$ ) is also shown. Similar trends as those observed in the case of $K=2$ are also seen here. We observe that here again as $N$ becomes large, $P_{o}$ approaches zero. It should be noted that when 


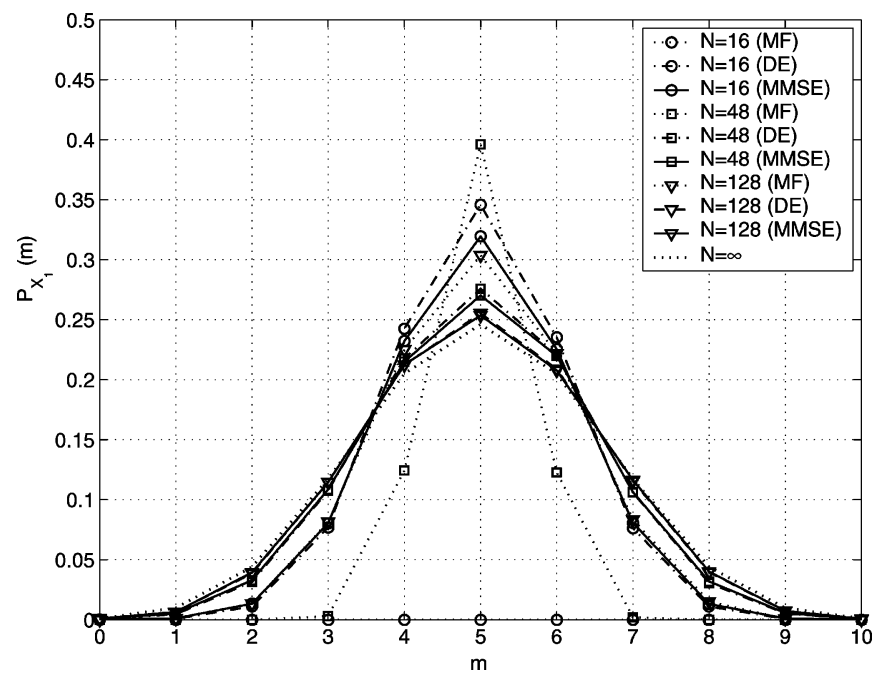

Fig. 8. The (pseudo) probability mass function of $X_{1}$ for different values of the processing gain $N$ for the ten-user two-carrier case (i.e., $D=2$ and $K=10$ ) for the $\mathrm{MF}$, the $\mathrm{DE}$, and the MMSE detector. $X_{1}$ is a random variable representing the number of users transmitting on the first carrier at Nash equilibrium.

$N$ is small, $\gamma^{*}$ cannot be achieved simultaneously by all the users at the output of the MFs. Therefore, users keep increasing their transmit powers, and hence no equilibrium is reached. This is the case until $N$ becomes large enough so that it can accommodate at least five users on each carrier (i.e., $N>25.6$ ). In practice, however, if $N$ is not large enough, some or all users end up transmitting at the maximum power.

In Fig. $8, P_{X_{1}}(m)$ is plotted as a function of $m$ for the MF, as well as the decorrelating and MMSE detectors for different values of $N$. The asymptotic approximation for $P_{X_{1}}(m)$, given by (33), is also shown. We see from the figure that as the processing gain increases, the pseudo PMF of $X_{1}$ becomes wider because the system becomes more interference-tolerant. Also, the equilibria for which the allocation of users to the carriers is highly asymmetric (e.g., $m=9$ and 10) are unlikely to happen. In other words, with a high probability, the users are evenly distributed between the two carriers. As expected, the pseudo PMFs of the DE and the MMSE detector are wider than those of the matched filer and are more closely approximated by (33), especially as $N$ becomes large. Based on the pseudo PMFs of $X_{1}$, we have plotted the standard deviation of $X_{1}$ for the MF, the DE, and the MMSE detector as a function of $N$ (see Fig. 9). It is observed that the standard deviation of $X_{1}$ increases as the processing gain increases and the values are higher for the multiuser detectors as compared with those of the MF. This means that it is more likely for the DE and the MMSE detector to have a more nonuniform distribution of users over the carriers at equilibrium. This, of course, makes sense because the multiuser detectors are more powerful in combating the multiple-access interference as compared with the MF.

We now investigate the effect of the number of carriers on the energy efficiency of the system. For a fixed bandwidth, as the number of carriers $D$ increases, the number of independent channels for each user increases. Hence, the quality of the "best" channel for each user improves. On the other hand, since

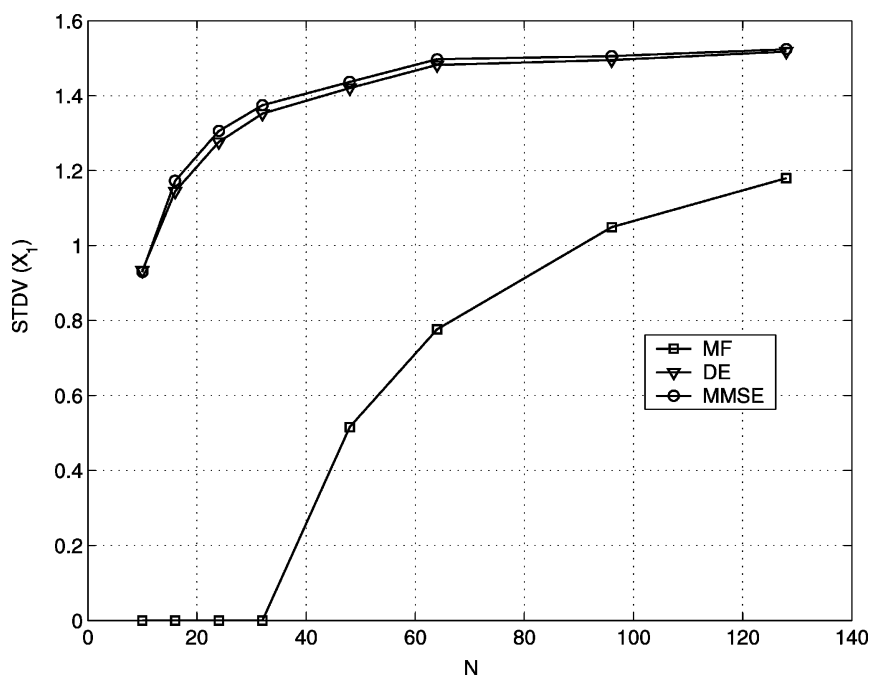

Fig. 9. The standard deviation for $X_{1}$ based on the pseudoprobability mass function as a function of the processing gain $N$ for the ten-user two carrier case (i.e., $D=2$ and $K=10$ ) for the MF, the DE, and the MMSE detector. $X_{1}$ is a random variable representing the number of users transmitting on the first carrier at Nash equilibrium.

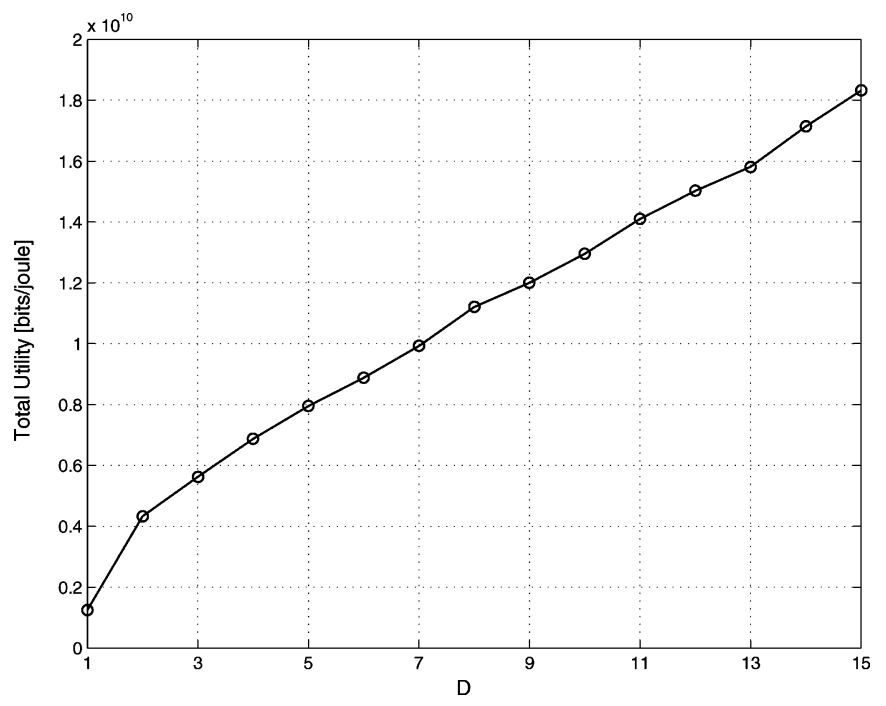

Fig. 10. Total utility versus number of carriers $D$ with 30 users for a fixed bandwidth. The processing gain is 256 when $D=1$ and decreasing proportionally as $D$ increases (i.e., $N=256 / D$ ).

the bandwidth is fixed, the processing gain for each carrier decreases as the number of carriers increases. Let us consider a system with 30 users $(K=30)$. We fix the bandwidth and change the number of carriers from 1 (i.e., single carrier) to 15. The processing gain for the case of $D=1$ is assumed to be 256. For each value of $D$, we run the BMP algorithm for 20 iterations and compute the total utility of the system at the end of the 20th iteration. We repeat this for 20000 channel realizations and average the results. Fig. 10 shows the average total utility versus number of carriers. It is observed from the figure that as the number of carriers increases, the total utility also increases. We might have expected the utility to decrease after a certain point because of the reduction in the processing gain. However, a smaller processing gain results in a more even distribution of users among the carrier. To demonstrate this, Fig. 11 shows the $P_{X_{1}}(m)$ as a function of $m$ for two-carrier case with $N=128$. 


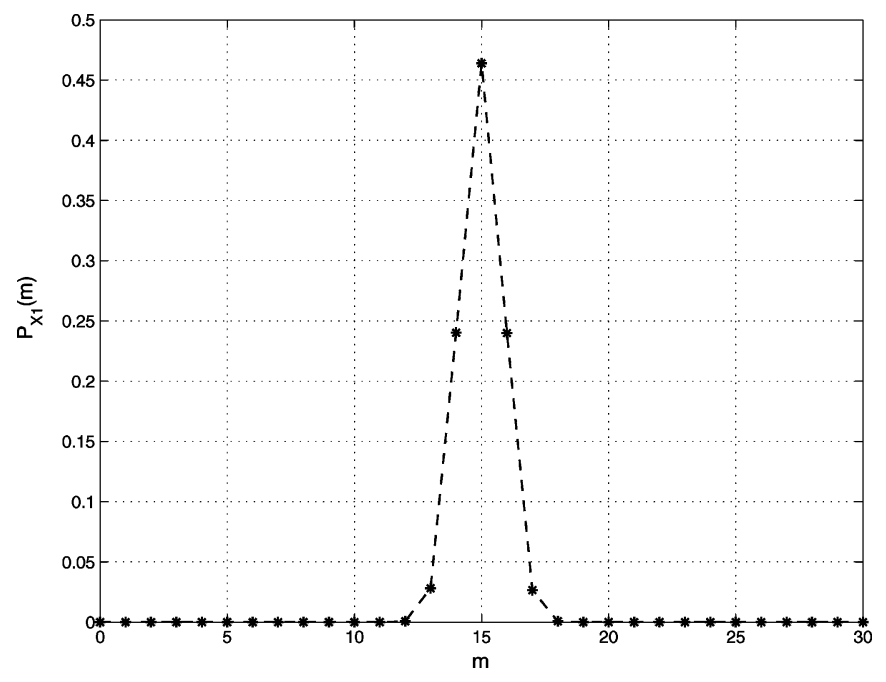

Fig. 11. Probability of having $m$ users on the first carrier at Nash equilibrium $P_{X_{1}}(m)$ for the 30-user, two-carrier case with processing gain equal to 128 (i.e., $D=2, K=30$, and $N=128$ ).

This plot is obtained by counting the number of users on each carrier at the end of the 20th iteration of the algorithm. It is seen that most of the time the users are equally distributed between the two carriers. Similarly, we can say that with $D$ carriers, most of the time, we will have $K / D$ users transmitting on each carrier. Therefore, the system does not suffer from excessive interference although the processing gain drops as $D$ increases. Since a system with a larger number of carriers benefits more from diversity, the total utility increases as the number of carriers increases.

We have also run simulations for the scenario in which the number of users and the number of carriers both increase but their ratio and the processing gain per carrier stay fixed. In particular, we considered the cases of $D=1,2,4$, and 8, with $N=$ 64 and $K / D=2$. For each case, we ran the BMP algorithm for 20000 channel realizations with MaxNumIter $=10 D$. For each channel realization, if a stable state were reached at the end of the last iteration, we would count the number of users on each carrier and record the values. While the results of the simulation are difficult to show graphically, we briefly describe some of the observed trends. We saw from the simulation that as $D$ increased, the probability that no Nash equilibrium existed also increased. On the other hand, asymmetric Nash equilibria became less likely. This is because as $D$ increases, the number of users also increases but the processing gain stays the same. Therefore, larger transmit powers are required to achieve $\gamma^{*}$ for asymmetric cases. As a result, nonuniform Nash equilibria become less probable.

We now compare our proposed approach, which jointly maximizes each user's utility over all the carriers, with the approach that maximizes each user's utility independently over each carrier. In the joint maximization approach, each user transmits only on the carrier that has the best effective channel, whereas in the other case, all users transmit on all the carriers such that the output SINR on each carrier is $\gamma^{*}$. We consider a system with two carriers and $N=128$. We fix $K$ and compute the sum of the utilities achieved by all users for 20000 channel realizations. The utility for each user is the ratio of the total

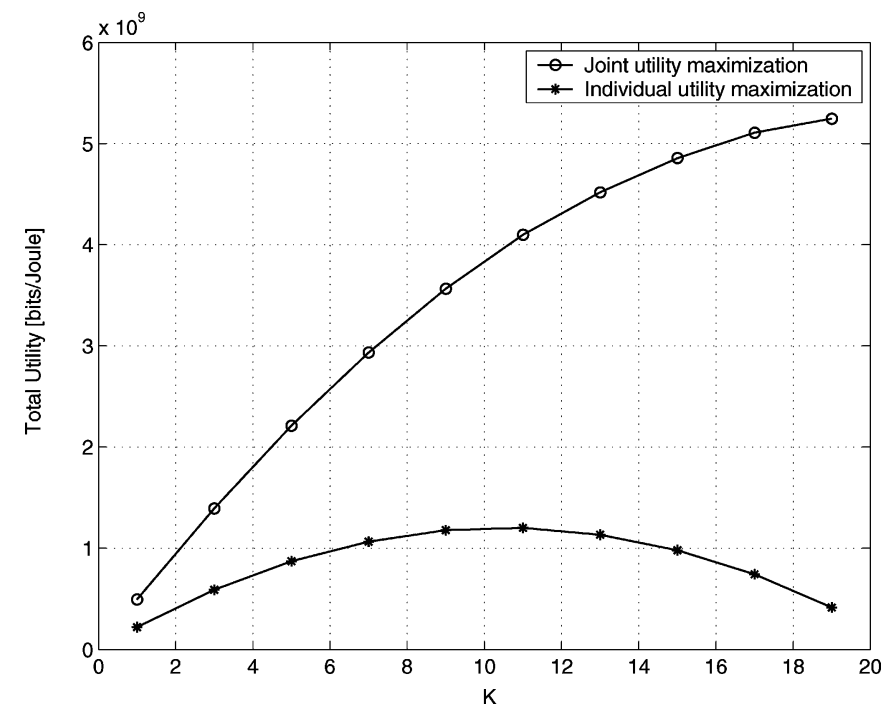

Fig. 12. Total utility versus number of users, $K$, for the two-carrier case with processing gain equal to 128 (i.e., $D=2$ and $N=128$ ).

number of transmitted bits over the two carriers to the total energy consumed. Fig. 12 shows the average total utility versus $K$ for the two approaches. We see a significant improvement in the achieved utility when joint maximization over all carriers is used. This is because when all the users transmit on every carrier, they cause unnecessary interference. To achieve $\gamma^{*}$, each user is hence forced to transmit at a higher power level which in turn results in a considerable reduction in the overall utility. In the joint optimization approach, each user transmits only on its "best" carrier. This way, the users perform a distributed interference avoidance mechanism which results in a higher overall utility.

\section{CONCLUSION}

We have modeled power control for multicarrier CDMA systems as a noncooperative game in which each user needs to decide how much power to transmit over each carrier to maximize its overall utility. The utility function has been defined as the overall throughput divided by the total transmit power over all the carriers and has units of bits per joule. This game is particularly difficult to analyze because users' strategies are vectors (rather than scalars) and the utility function is not a quasi-concave function of the user's strategy. For this utility function, we have shown that at a Nash equilibrium each user transmits only on the carrier that has the best "effective channel" for that user. In addition, we have derived conditions for existence and uniqueness of Nash equilibrium, and we have characterized the distribution of the users over the carriers at equilibrium. Basically, satisfaction of a set of inequalities by the relative channel gains of each user is sufficient for the existence of a Nash equilibrium. We have also proposed an iterative and distributed algorithm for reaching the equilibrium (when it exists). We have shown that our proposed approach results in a significant improvement in the total network utility achieved at equilibrium compared with a single-carrier system and also to a multicarrier system in which users maximize their utilities over each carrier independently. 


\section{APPENDIX I \\ Proof of CONVERGENCE OF THE BMP ALGORITHM FOR THE TWO-USER TWO-CARRIER CASE}

We prove that for the case of two users and two carriers, the BMP algorithm converges to an equilibrium whenever it exists. For the sake of simplicity, here we assume a MF receiver. However, the proof can easily be generalized to other linear receivers. Because of symmetry, we need only to consider two of the four possible Nash equilibria, namely, the equilibrium in which both users transmit on the first carrier, denoted by (12, ), and the one in which user 1 transmits on the first carrier and user 2 transmits on the second carrier, denoted by $(1,2)$.

\section{A. The Case in Which (12, ) is the Nash Equilibrium}

In this case, we have $h_{11} / h_{12}>\Theta_{2}$ and $h_{21} / h_{22}>\Theta_{2}$. The received powers at equilibrium are given by $q_{11}^{*}=p_{11}^{*} h_{11}=$ $\gamma^{*} \sigma^{2} \Theta_{2}$ and $q_{21}^{*}=p_{21}^{*} h_{21}=\gamma^{*} \sigma^{2} \Theta_{2}$.

1) Starting From (12, ): If user 1 (user 2) starts first and the received power for user 2 (user 1 ) is less than $\gamma^{*} \sigma^{2} \Theta_{2}$, user 1 (user 2) stays on the first carrier with its updated received power being less than $\gamma^{*} \sigma^{2} \Theta_{2}$ but larger than that of user 2 (user 1). Since the received powers of both users are less than $\gamma^{*} \sigma^{2} \Theta_{2}$, user 1 and user 2 both continue to stay on the first carrier and update their powers until the equilibrium is reached.

If the received powers are such that user 1 (user 2) jumps to the second carrier, then user 2 (user 1) stays on the first carrier with its received power equal to $\gamma^{*} \sigma^{2}$. Now, because $h_{11} / h_{12}>$ $\Theta_{2}\left(h_{21} / h_{22}>\Theta_{2}\right)$, user 1 (user 2) jumps back to the first carrier with $q_{11}=\gamma^{*} \sigma^{2} / \Theta_{0}\left(q_{21}=\gamma^{*} \sigma^{2} / \Theta_{0}\right)$ which is less than $\gamma^{*} \sigma^{2} \Theta_{2}$. From this point, both users stay on the first carrier and update their powers until the equilibrium is reached.

2) Starting From ( , 12): Since $h_{11} / h_{12}>\Theta_{2}\left(h_{21} / h_{22}>\right.$ $\Theta_{2}$ ), if user 1 (user 2) starts first, it will jump to carrier one with its received power equal to $\gamma^{*} \sigma^{2}$. Now, user 2 (user 1) jumps to carrier one with $q_{21}=\gamma^{*} \sigma^{2} / \Theta_{0}\left(q_{11}=\gamma^{*} \sigma^{2} / \Theta_{0}\right)$ which is less than $\gamma^{*} \sigma^{2} \Theta_{2}$. From this point, both users stay on the first carrier and update their powers until the equilibrium is reached.

3) Starting From $(1,2)$ : If user 1 starts first, it will stay on the first carrier with $q_{11}=\gamma^{*} \sigma^{2}$. Then, user 2 will jump on first carrier with $q_{21}=\gamma^{*} \sigma^{2} / \Theta_{0}$. Therefore, as before, both users stay on the first carrier and update their powers until the equilibrium is reached.

If user 2 starts first and stays on the second carrier, then we are back to the case that was just described. If user 2 jumps to the first carrier and user 1 stays on the first carrier, then we are back to the case that was already explained in Appendix I-A1. On the other hand, if user 2 jumps to the first carrier and user 1 jumps to the second carrier, then user 2 will stay on the first carrier with $q_{21}=\gamma^{*} \sigma^{2}$. After this, user 1 jumps back to the first carrier with $q_{11}=\gamma^{*} \sigma^{2} / \Theta_{0}$. Thus, again, both users stay on the first carrier and update their powers until the equilibrium is reached.

4) Starting From $(2,1)$ : The argument is similar to Appendix I-A3 due to symmetry.

\section{B. The Case in Which $(1,2)$ is the Nash Equilibrium}

Let us consider the case in which $(1,2)$ is the only Nash equilibrium. This corresponds to $h_{11} / h_{12}>\Theta_{0}$ and $h_{21} / h_{22}<\Theta_{0}$ or $h_{11} / h_{12}>1 / \Theta_{0}$ and $h_{21} / h_{22}<1 / \Theta_{0}$. For this equilibrium, we have $q_{11}^{*}=q_{22}^{*}=\gamma^{*} \sigma^{2}$.

1) Starting From (12, ): If user 1 starts first and stays on the first carrier, then user 2 will jump to carrier two with $q_{22}=$ $\gamma^{*} \sigma^{2}$. As a result, user 1 will stay on the first carrier with $q_{11}^{*}=$ $\gamma^{*} \sigma^{2}$, and hence the equilibrium is reached.

If user 1 starts first but jumps on the second carrier, then because we are assuming that $(1,2)$ is the only Nash equilibrium (i.e., $h_{11} / h_{12}>\Theta_{0}$ and $h_{21} / h_{22}<\Theta_{0}$ or $h_{11} / h_{12}>1 / \Theta_{0}$ and $h_{21} / h_{22}<1 / \Theta_{0}$ ), user 2 will also jump on the second carrier. As a result, user 1 will jump back to carrier one with $q_{11}^{*}=\gamma^{*} \sigma^{2}$ and user 2 will stay on carrier two with $q_{22}^{*}=\gamma^{*} \sigma^{2}$. Hence, the equilibrium is reached.

If user 2 starts first, it will jump on the second carrier with $q_{22}=\gamma^{*} \sigma^{2}$. As a result, user 1 will stay on the first carrier with $q_{11}^{*}=\gamma^{*} \sigma^{2}$, and hence equilibrium is reached.

2) Starting From $(, 12)$ : The argument is similar to Appendix I-B1 due to symmetry.

3) Starting From $(1,2)$ : If user 1 (user 2) starts first, it will stay on the first ( second) carrier with $q_{11}=\gamma^{*} \sigma^{2}\left(q_{22}=\gamma^{*} \sigma^{2}\right)$. As a result, user 1 (user 2) will stay on the first (second) carrier, and hence equilibrium is reached.

4) Starting From $(2,1)$ ): If user 1 (user 2) starts first and jumps to the first (second) carrier, user 2 (user 1) will jump to the second (first) carrier with $q_{22}=\gamma^{*} \sigma^{2}\left(q_{11}=\gamma^{*} \sigma^{2}\right)$. Thus, user 1 (user 2) will stay on the first (second) carrier and the equilibrium is reached.

If user 1 (user 2) starts first but it stays on the second (first) carrier, then we will have $q_{12}=\gamma^{*} \sigma^{2}\left(q_{21}=\gamma^{*} \sigma^{2}\right)$. But because we are assuming that $(1,2)$ is the only Nash equilibrium (i.e., $h_{11} / h_{12}>\Theta_{0}$ and $h_{21} / h_{22}<\Theta_{0}$ or $h_{11} / h_{12}>1 / \Theta_{0}$ and $h_{21} / h_{22}<1 / \Theta_{0}$ ), user 2 (user 1 ) jumps to carrier two (one) with $q_{22}^{*}=\gamma^{*} \sigma^{2} / \Theta_{0}\left(q_{22}^{*}=\gamma^{*} \sigma^{2} / \Theta_{0}\right)$. As a result, user 1 (user 2) jumps to the first (second) carrier with $q_{11}^{*}=\gamma^{*} \sigma^{2}$ $\left(q_{22}^{*}=\gamma^{*} \sigma^{2}\right)$. Thus, user 2 (user 1) will stay on the second (first) carrier and the equilibrium is reached.

It can be seen from the above arguments that if $(1,2)$ and $(2,1)$ are both Nash equilibria, the algorithm converges to one of them depending on the initial condition.

\section{APPENDIX II \\ ProOf OF CONVERGENCE OF THE BMP AlgorithM FOR THE TWO-USER D-CARRIER CASE}

We prove the convergence of the BMP algorithm for the case of two users and three carriers. Generalization to the two-user $D$-carrier case is straightforward. In the two-user three-carrier case, there are nine possible Nash equilibria. For the sake of simplicity, we assume a MF receiver. However, the proof can easily be generalized to other linear receivers. Here, we focus on the $(12,$,$) Nash equilibrium. An argument similar to the$ one given here and the ones used for the two-user two-carrier case can be used for other equilibria. 


\section{A. The Case in Which $(12$, , ) is the Nash Equilibrium}

In this case, we have $h_{11} / h_{12}>\Theta_{2}, h_{11} / h_{13}>\Theta_{2}$, $h_{21} / h_{22}>\Theta_{2}$, and $h_{21} / h_{23}>\Theta_{2}$. The received powers at equilibrium are given by $q_{11}^{*}=p_{11}^{*} h_{11}=\gamma^{*} \sigma^{2} \Theta_{2}$ and $q_{21}^{*}=p_{21}^{*} h_{21}=\gamma^{*} \sigma^{2} \Theta_{2}$.

1) Starting From (12, , ): If user 1 (user 2) starts first and the received power for user 2 (user 1) is less than $\gamma^{*} \sigma^{2} \Theta_{2}$, user 1 (user 2) stays on the first carrier with its updated received power being less than $\gamma^{*} \sigma^{2} \Theta_{2}$ but larger than that of user 1 (user 2). Since the received powers of both users are less than $\gamma^{*} \sigma^{2} \Theta_{2}$, user 1 and user 2 both continue to stay on the first carrier and update their powers until the equilibrium is reached.

If the received powers are such that user 1 (user 2) jumps to the second or third carrier, then user 2 (user 1) stays on the first carrier with its received power equal to $\gamma^{*} \sigma^{2}$. Now, because $h_{11} / h_{12}>\Theta_{2}, h_{11} / h_{13}>\Theta_{2}, h_{21} / h_{22}>\Theta_{2}$, and $h_{21} / h_{23}>$ $\Theta_{2}$, user 1 (user 2) jumps back to the first carrier with $q_{11}=$ $\gamma^{*} \sigma^{2} / \Theta_{0}\left(q_{21}=\gamma^{*} \sigma^{2} / \Theta_{0}\right)$ which is less than $\gamma^{*} \sigma^{2} \Theta_{2}$. From this point, both users stay on the first carrier and update their powers until the equilibrium is reached.

2) Starting From (, 12, ): Since $h_{11} / h_{12}>\Theta_{2}$ $\left(h_{21} / h_{22}>\Theta_{2}\right)$, if user 1 (user 2) starts first, it will jump to carrier one with its received power equal to $\gamma^{*} \sigma^{2}$. Now, user 2 (user 1) jumps to carrier one with $q_{21}=\gamma^{*} \sigma^{2} / \Theta_{0}$ $\left(q_{11}=\gamma^{*} \sigma^{2} / \Theta_{0}\right)$ which is less than $\gamma^{*} \sigma^{2} \Theta_{2}$. From this point, both users stay on the first carrier and update their powers until the equilibrium is reached.

3) Starting From ( , , 12): The argument is similar to Appendix II-A2 due to symmetry.

4) Starting From (1, 2, ): If user 1 starts first, it will stay on the first carrier with $q_{11}=\gamma^{*} \sigma^{2}$. Then, user 2 will jump on first carrier with $q_{21}=\gamma^{*} \sigma^{2} / \Theta_{0}$. Therefore, as before, both users stay on the first carrier and update their powers until the equilibrium is reached.

If user 2 starts first and stays on the second carrier, then we are back to the case that was just described. If user 2 jumps to the third carrier, user 1 stays on the first carrier with $q_{11}=\gamma^{*} \sigma^{2}$. Then, user 2 jumps to the first carrier and both users stay there until equilibrium is reached.

If user 2 jumps to the first carrier and user 1 stays on the first carrier, then we are back to the case that was already explained in Appendix II-A1. On the other hand, if user 2 jumps to the first carrier and user 1 jumps to the second or third carrier, then user 2 will stay on the first carrier with $q_{21}=\gamma^{*} \sigma^{2}$. After this, user 1 jumps back to the first carrier with $q_{11}=\gamma^{*} \sigma^{2} / \Theta_{0}$. Thus, again, both users stay on the first carrier and update their powers until the equilibrium is reached.

5) Starting From $(1,2)$ : The argument is similar to Appendix II-A4 due to symmetry.

6) Starting From $(2,1$,$) : The argument is similar to$ Appendix II-A4 due to symmetry.

7) Starting From $(2,, 1)$ : The argument is similar to Appendix II-A4 due to symmetry.

8) Starting From ( , 1, 2): If user 1 (user 2) starts first, it will jump to carrier one with $q_{11}=\gamma^{*} \sigma^{2}\left(q_{21}=\gamma^{*} \sigma^{2}\right)$. Then, user 2 (user 1) jumps to the first carrier. Both user will then stay on the first carrier until equilibrium is reached.

9) Starting From $(, 2,1)$ : The argument is similar to Appendix II-A8 due to symmetry.

\section{APPENDIX III}

\section{PROOF OF CONVERGENCE OF THE BMP ALGORITHM FOR THE THREE-USER TWO-CARRIER CASE}

We prove the convergence of the BMP algorithm for the case of three users and two carriers. In this case, there are 27 possible Nash equilibria. Here, we focus on the $(123, \quad)$ and $(12,3)$ Nash equilibria. Similar arguments can be used for other equilibria.

\section{A. The Case in Which (123, ) is the Nash Equilibrium}

In this case, we have $h_{11} / h_{12}>\Theta_{3}, h_{21} / h_{22}>\Theta_{3}$, and $h_{31} / h_{32}>\Theta_{3}$. The received powers at equilibrium are given by $q_{11}^{*}=q_{21}^{*}=q_{31}^{*}=\gamma^{*} \sigma^{2} \Theta_{3}$. Here, we only discuss the convergence for two possible starting points. Similar arguments can be applied to the other cases as well.

1) Starting From (123, ): If the powers are such that the three users stay on the first carrier, then they keep updating their powers until they reach the equilibrium.

On the other hand, the powers can be such that one of the users jumps to the second carrier. Without loss of generality, let us assume that user 3 jumps to the second carrier, i.e., let us assume that we have reached $(12,3)$ and user 1 has to now update its power.

- Now, if user 1 also jumps to the second carrier, user 2 stays on the first carrier with $q_{21}=\gamma^{*} \sigma^{2}$. As a result, user 3 jumps back to carrier one with $q_{31}=\gamma^{*} \sigma^{2} / \Theta_{0}$. Thus, user 1 also jumps to the first carrier and the users stay there until the equilibrium is reached.

- Now, let us assume that user 3 jumps but user 1 stays.

- If $q_{21} \leq \gamma^{*} \sigma^{2} \Theta_{3}$, then after user 1 updates its power, we will have $q_{11} \leq \gamma^{*} \sigma^{2} \Theta_{3}$. Therefore, user 1 and 2 both stay on the first carrier and user 3 also jumps back to carrier one. The three users stay on this carrier until equilibrium is reached.

- If $q_{21}>\gamma^{*} \sigma^{2} \Theta_{3}$, then after user 1 updates its power, we will have $q_{11}<q_{12}$. If user 2 stays, then user 1 will also stay. In this case, the powers of users 1 and 2 decrease until user 3 jumps back to the first carrier. In this case, $q_{31} \leq \max \left\{q_{11}, q_{21}\right\}$. One or two users may still jump to the second carrier but the powers of the users on the first carrier keep decreasing. As a result, the users will eventually come back to the first carrier and at some point, the powers become such that all three users stay on the first carrier until the equilibrium is reached. On the other hand, if user 2 jumps to the second carrier and user 3 stays on the second carrier, then user 1 stays on the first carrier with $q_{11} \leq \gamma^{*} \sigma^{2}$. As a result, users 2 and 3 jump back to the first carrier and all three users stay there until an equilibrium is reached. In the case where user 2 jumps on the second carrier but user 3 jumps back to the first carrier, we will have $q_{31}<q_{11}$. One or two may still continue to jump to the second carrier but the powers of the users on the first carrier keep decreasing. Eventually, a point is reached where all three users come back to the first carrier and stay there until the equilibrium is reached.

2) Starting From $(1,23)$ : If user 1 starts first, it will stay on the first carrier. As a result, users 2 and 3 will also jump to the 
first carrier and all three users stay there until the equilibrium is reached.

Now, consider the case where user 2 starts first.

- If user 2 jumps on the first carrier and user 3 also jumps then we are back to the case that was discussed in Appendix III-A1. If user 2 jumps but user 3 stays on second carrier, then we are back to the $(12,3)$ case discussed as part of Appendix III-A1.

- If user 2 stays on the second carrier and user 3 also stays, then user 1 will stay on the first carrier with $q_{11}=\gamma^{*} \sigma^{2}$. As a result, users 2 and 3 will jump to the first carrier and all three users stay there until an equilibrium is reached. On the other hand, if user 2 stays on the second carrier but user 3 jumps to the first carrier, then we will have the $(13,2)$ case. Although the users may still take turn in jumping to the second carrier, the powers of the users on the first carrier keep decreasing. Eventually, a point is reached where all three users come back to the first carrier and stay there until the equilibrium is reached. This is similar to the $(12,3)$ scenario discussed as part of Appendix III-A1.

Let us now consider the case in which user 3 starts first.

- If user 3 stays on the second carrier, then user 1 will stay on the first carrier with $q_{11}=\gamma^{*} \sigma^{2}$. As a result, users 2 and 3 will jump to the first carrier and all three users stay there until the equilibrium is reached.

- If user 3 jumps to the first carrier, then we will be in the $(13,2)$ case which is similar in nature to the he $(12,3)$ scenario discussed as part of Appendix III-A1.

Similar arguments can be used for the remaining 25 starting points. It should be noted that symmetry can be used to reduce the number of cases that need to be considered.

\section{B. The Case in Which $(12,3)$ is the Nash Equilibrium}

Let us consider the case of $h_{11} / h_{12}>\Theta_{2} / \Theta_{0}, h_{21} / h_{22}>$ $\Theta_{2} / \Theta_{0}$ and $h_{31} / h_{32}<\Theta_{2} / \Theta_{0}$ for which $(12,3)$ is the only equilibrium. Other cases can be treated similarly. For the sake of brevity, we only discuss the $(3,12)$ starting point. Similar arguments can be used for other starting points.

1) Starting From $(3,12)$ : Let us now consider the case in which user 1 starts first.

- If user 1 jumps to the first carrier and user 2 also jumps, then user 3 will jump to the second carrier with $q_{32}=$ $\gamma^{*} \sigma^{2}$. Now, if either user 1 or user 2 jumps to the second carrier, user 3 still stays in the second carrier because $h_{31} / h_{32}<\Theta_{2} / \Theta_{0}$. Therefore, users 1 and 2 will eventually go back to the first carrier and stay there until the equilibrium is reached. On the other hand, if user 1 jumps to the first carrier but user 2 stays on the second carrier, then user 3 will jump to the second carrier with $q_{32}=$ $\gamma^{*} \sigma^{2} / \Theta_{0}$. As a result, user 1 stays on the first carrier with $q_{11}=\gamma^{*} \sigma^{2}$ and user 2 also jumps to the first carrier. They stay there until the equilibrium is reached.

- Now, consider the case where user 1 stays on the second carrier and user 2 also stays.

- If user 3 jumps to the second carrier, then users 1 and 2 jump to the first carrier and user 3 will stay on the second carrier. The users stay there until the equilibrium is reached.

- If user 3 stays on the first carrier, then we will have $q_{31}=\gamma^{*} \sigma^{2}$. As a result, users 1 and 2 jump to the first carrier and then user 3 jumps to the second carrier. The users stay there until the equilibrium is reached.

Let us now consider the case in which user 2 starts first.

- If user 2 stays on the second carrier and user 3 stays on the first carrier, then user 1 jumps on the first carrier with $q_{11}=\gamma^{*} \sigma^{2} / \Theta_{0}$. As a result, user 2 also jumps to the first carrier, and consequently user 3 jumps to the second carrier. The users stay there until the equilibrium is reached. On the other hand, if user 2 stays on the second carrier and user 3 jumps to the second carrier, then users 1 and 2 will jump to the first carrier. As a result, user 3 stays on the second carrier. Users 1 and 2 will stay on the first carrier until the equilibrium is reached.

- Now, assume that user 2 jumps to the first carrier.

- If user 3 stays on the first carrier and user 1 jumps to the first carrier, then independent of whether user 2 jumps to the second carrier, user 3 will jump to the second carrier. Because of this, users 1 and 2 will eventually jump to the first carrier and stay there until the equilibrium is reached. On the other hand, if user 3 stays on the first carrier and user 1 stays on the second carrier, then by going through the two possibilities for user 2, it can be shown that user 3 will eventually jump to the second carrier and users 1 and 2 will jump to the first carrier. The users then stay there until the equilibrium is reached.

- If user 3 jumps to the second carrier and user 1 stays on the second carrier, then user 2 will stay on the first carrier with $q_{21}=\gamma^{*} \sigma^{2}$. It can be shown by going through the two possibilities for user 3 , that users 1 and 2 will eventually end up on the first carrier and user 3 will end up on the second carrier. The users stay there until the equilibrium is reached.

Let us now consider the case in which user 3 starts first. If user 3 stays on the first carrier, then users 1 and 2 will also jump to the first carrier. As a result, user 3 will jump to the second carrier. The users stay there until the equilibrium is reached. On the other hand, if user 3 jumps to the second carrier, then users 1 and 2 will jump to the first carrier and user 3 will then stay on the second carrier. The users stay there until the equilibrium is reached.

The convergence for other cases can be verified using similar arguments.

\section{REFERENCES}

[1] A. B. MacKenzie and S. B. Wicker, "Game theory in communications: Motivation, explanation, and application to power control," in Proc. IEEE Global Telecommun. Conf., San Antonio, TX, Nov. 2001, pp. 821-826.

[2] D. J. Goodman and N. B. Mandayam, "Power control for wireless data," IEEE Pers. Commun., vol. 7, pp. 48-54, Apr. 2000.

[3] H. Ji and C.-Y. Huang, "Non-cooperative uplink power control in cellular radio systems," Wireless Netw., vol. 4, pp. 233-240, Apr. 1998.

[4] C. U. Saraydar, N. B. Mandayam, and D. J. Goodman, "Efficient power control via pricing in wireless data networks," IEEE Trans. Commun., vol. 50, pp. 291-303, Feb. 2002. 
[5] M. Xiao, N. B. Shroff, and E. K. P. Chong, "A utility-based power-control scheme in wireless cellular systems," IEEE/ACM Trans. Netw., vol. 11, pp. 210-221, Apr. 2003.

[6] C. Zhou, P. Zhang, M. L. Honig, and S. Jordan, "Two-cell power allocation for downlink CDMA," IEEE Trans. Wireless Commun., vol. 3, pp. 2256-2266, Nov. 2004

[7] T. Alpcan, T. Basar, R. Srikant, and E. Altman, "CDMA uplink power control as a noncooperative game," Wireless Netw., vol. 8, pp. 659-669, Nov. 2002.

[8] C. W. Sung and W. S. Wong, "A noncooperative power control game for multirate CDMA data networks," IEEE Trans. Wireless Commun., vol. 2, pp. 186-194, Jan. 2003.

[9] F. Meshkati, H. V. Poor, S. C. Schwartz, and N. B. Mandayam, "An energy-efficient approach to power control and receiver design in wireless data networks," IEEE Trans. Commun., vol. 53, pp. 1885-1894, Nov. 2005

[10] S. Hara and R. Prasad, "Overview of multicarrier CDMA," IEEE Commun. Mag., pp. 126-133, Dec. 1997.

[11] V. M. DaSilva and E. S. Sousa, "Performance of orthogonal CDMA codes for quasi-synchronous communication systems," in Proc. 2nd IEEE Int. Conf. Universal Pers. Commun., Ottawa, Canada, Jun. 1993, pp. 995-999.

[12] T. M. Cover and J. A. Thomas, Elements of Information Theory. New York: Wiley, 1991.

[13] S. Vishwanath, S. A. Jafar, and A. Goldsmith, "Adaptive resource allocation in composite fading channels," in Proc. IEEE Global Telecommun. Conf., San Antonio, TX, Nov. 2001, pp. 1312-1316.

[14] G. Munz, S. Pfletschinger, and J. Speidel, "An efficient waterfilling algorithm for multiple access OFDM," in Proc. IEEE Global Telecommun. Conf., Taipei, Taiwan, Nov. 2002, pp. 681-685.

[15] Z. Shen, J. G. Andrews, and B. L. Evans, "Optimal power allocation in multiuser OFDM systems," in Proc. IEEE Global Telecommun. Conf., San Francisco, CA, Dec. 2003, pp. 337-341.

[16] D. Fudenberg and J. Tirole, Game Theory. Cambridge, MA: MIT Press, 1991

[17] V. Rodriguez, "An analytical foundation for resource management in wireless communication," in Proc. IEEE Global Telecommun. Conf., San Francisco, CA, Dec. 2003, pp. 898-902.

[18] W. Yu, G. Ginis, and J. M. Cioffi, "Distributed multiuser power control for digital subscriber lines," IEEE J. Sel. Areas Commun., vol. 20, pp. 1105-1115, Jun. 2002

[19] R. Lupas and S. Verdú, "Linear multiuser detectors for synchronous code-division multiple access channels," IEEE Trans. Inf. Theory, vol. 35, pp. 123-136, Jan. 1989

[20] U. Madhow and M. L. Honig, "MMSE interference suppression for direct-sequence spread-spectrum CDMA," IEEE Trans. Commun., vol. 42, pp. 3178-3188, Dec. 1994.

[21] P. Hande, S. Rangan, and M. Chiang, "Distributed power control for optimal SIR assignment in wireless data networks," in Proc. IEEE INFOCOM, Barcelona, Spain, Apr. 2006

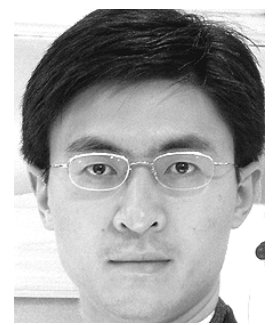

Mung Chiang (S'00-M'03) received the B.S (Hon.) degree in electrical engineering and mathematics, and the M.S. and Ph.D. degrees in electrical engineering from Stanford University, Stanford, CA

$\mathrm{He}$ is an Assistant Professor of Electrical Engineering at Princeton University, Princeton, NJ. His research interests include nonlinear optimization of communication systems, distributed algorithms and network protocols, architectures and algorithms in broadband access networks, and information theoretic limits of data transmission and compression.

Dr. Chiang has been awarded as a Hertz Foundation Fellow and received the Stanford University School of Engineering Terman Award, the SBC Communications New Technology Introduction Contribution Award, the National Science Foundation CAREER Award, and the Princeton University Howard B. Wentz Junior Faculty Award. He is the Lead Guest Editor of the IEEE JOURNAL ON SELECTED AREAS In COMMUNICATIONS (Special Issue on Nonlinear Optimization of Communication Systems), a Guest Editor of the IEEE TRANSACTIONS ON INFORMATION THEORY and the IEEE/ACM TRANSACTIONS ON NETWORKING (Joint Special Issue on Networking and Information Theory), and the Program Co-Chair of the 38th Conference on Information Sciences and Systems.

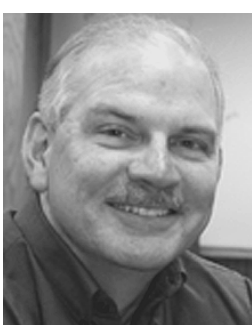

H. Vincent Poor (S'72-M'77-SM'82-F'87) received the Ph.D. degree in electrical engineering and computer science from Princeton University, Princeton, NJ, in 1977.

From 1977 to 1990 , he was on the faculty of the University of Illinois at Urbana-Champaign, Urbana. Since 1990, he has been on the faculty at Princeton University, where he is Michael Henry Strater University Professor of Electrical Engineering and Dean of the School of Engineering and Applied Science. His research interests are in the areas of statistical signal processing and its applications in wireless networks and related fields. Among his publications in these areas is the recent book Wireless Networks: Multiuser Detection in Cross-Layer Design (Springer: New York, 2005)

Dr. Poor is a member of the National Academy of Engineering and the American Academy of Arts and Sciences, and is a Fellow of the Institute of Mathematical Statistics, the Optical Society of America, and other organizations. Recent recognition of his work includes the Joint Paper Award of the IEEE Communications and Information Theory Societies (2001), the NSF Director's Award for Distinguished Teaching Scholars (2002), a Guggenheim Fellowship (2002-2003), and the IEEE Education Medal (2005). In 1990, he served as President of the IEEE Information Theory Society, and from 1991 to 1992, he was a member of the IEEE Board of Directors. He is currently serving as the Editor-in-Chief of the IEEE TRANSACTIONS ON INFORMATION THEORY.

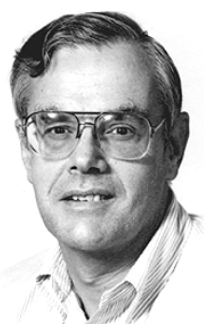

Stuart C. Schwartz (S'64-M'66-SM'83-F'92LF'04) received the B.S. and M.S. degrees from the Massachusetts Institute of Technology (MIT), Cambridge, in 1961, and the Ph.D. degree from the University of Michigan, Ann Arbor, in 1966.

While at MIT, he was associated with the Naval Supersonic Laboratory and the Instrumentation Laboratory (now the Draper Laboratories). During 1961-1962, he was at the Jet Propulsion Laboratory, Pasadena, CA, working on problems in orbit estimation and telemetry. During the academic year 1980-1981, he was a member of the technical staff at the Radio Research Laboratory, Bell Telephone Laboratories, Crawford Hill, NJ, working in the area of mobile telephony. He is currently a Professor of Electrical Engineering at Princeton University, Princeton, NJ. He was chair of the department during the period 1985-1994, and served as Associate Dean for the School of Engineering during the period July 1977-June 1980. During the academic year 1972-1973, he was a John S. Guggenheim Fellow and Visiting Associate Professor at the Department of Electrical Engineering, Technion, Haifa, Israel. He has also held visiting academic appointments at Dartmouth, University of California, Berkeley, and the Image Sciences Laboratory, ETH, Zurich. His principal research interests are in statistical communication theory, signal and image processing. 